\title{
Nonlinear energy transfer to short gravity waves in the presence of long waves
}

\author{
By H. S. ÖLMEZ† AND J. H. MILGRAM \\ Department of Ocean Engineering, Massachusetts Institute of Technology, \\ Cambridge, MA 02139, USA
}

(Received 26 April 1994 and in revised form 29 November 1994)

Existing theories for calculating the energy transfer rates to gravity waves due to resonant nonlinear interactions among wave components whose lengths are long in comparison to wave elevations have been verified experimentally and are well accepted. There is uncertainty, however, about prediction of energy transfer rates within a set of waves having short to moderate lengths when these are present simultaneously with a long wave whose amplitude is not small in comparison to the short wavelengths. Here we implement both a direct numerical method that avoids small-amplitude approximations and a spectral method which includes perturbations of high order. These are applied to an interacting set of short- to intermediate-length waves with and without the presence of a large long wave. The same cases are also studied experimentally. Experimentally and numerical results are in reasonable agreement with the finding that the long wave does influence the energy transfer rates. The physical reason for this is identified and the implications for computations of energy transfer to short waves in a wave spectrum are discussed.

\section{Introduction}

In recent years, there has been increased interest in the energy balance of short sea waves because of the influence of these waves on microwave remote sensing of the sea surface. One component of the energy balance is the nonlinear energy transfer among differing wavenumbers.

It is well accepted that energy transfer in the energy-containing portion of a wave spectrum can be calculated by the method pioneered by Hasselmann (1962). This is a stochastic treatment of fundamental interactions between groups of four waves first studied theoretically by Phillips (1960). Both the fundamental theory and the stochastic application are based on a perturbation theory which presumes that wave amplitudes are small in comparison to wavelengths. This presumption is reasonably well satisfied by waves whose lengths and frequencies are close to those at the spectral peak. Most applications of the Hasselmann theory have been for waves whose frequencies are less than 2.5 times the spectral peak frequency. For typical spectral energy distributions, most of the energy transfer at a specified frequency comes from quartet interactions of waves in a relatively small frequency range surrounding the specified frequency (Hasselmann \& Hasselmann 1985).

When short sea waves are considered, they generally exist in the presence of long waves whose amplitudes are not small in comparison to the short wavelengths. Two questions arise.

$\dagger$ Present address: Bilkent University, Faculty of Business Administration, Bilkent 06533, Ankara, Turkey. 

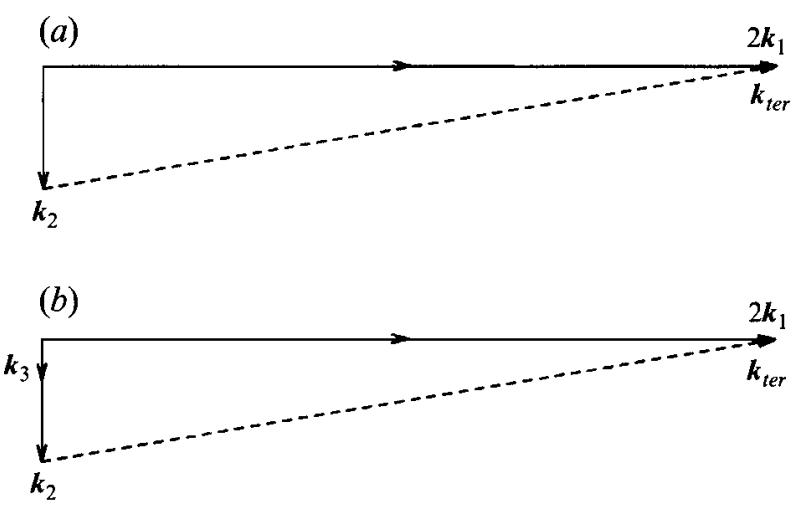

FIGURE 1. (a) Wave configuration for the special triad. (b) Wave configuration for the special triad in the presence of an underlying long wave.

(i) To what extent is the nonlinear energy transfer to (or from) short waves due to interactions with waves with relatively similar frequencies altered by the presence of large waves with much lower frequencies and correspondingly longer lengths?

(ii) To what extent are the short-wave energy transfer results of computation methods based on small-amplitude expansions compromised by the presence of long waves?

In this paper we have answered these questions, in part, through computations and experiments on a set of interacting wave components both with and without the presence of an additional long large wave. The numerical studies comprise two approaches.

Our first approach is a direct numerical method which avoids perturbation expansions altogether. A time-stepping scheme is used to trace the evolution of water waves in a bounded domain. With prescribed initial conditions, the fully nonlinear boundary conditions are time-marched to determine the free-surface elevation and velocity potential in time. This requires knowledge of the normal velocity on the freesurface whose computation is numerically intensive and requires considerable computational resources. Our direct approach to obtain the normal velocity is to apply Green's theorem using the Rankine source Green function and to solve the resulting integral equation at each time step.

The second numerical approach is a spectral method which utilizes small-amplitude expansions, but which includes perturbations of arbitrarily high order. It is along the lines of the spectral methods initially developed and used for other problems by Dommermuth \& Yue (1987) and West et al. (1987). By comparing results from the two approaches, the accuracy of using the expansions is assessed.

Experimental confirmation exists for the theory of the growth of a short wave due to nonlinear interactions amongst intermediate-length waves in the absence of a large long wave (Longuet-Higgins \& Smith 1966; McGoldrick et al. 1966). These experiments clearly showed the energy transfer and confirmed the initial growth rates given by the classical perturbation theory. Later Tomita (1989) repeated the experiments in a much larger wave tank to quantify the long-term evolution of the interaction. None of these experiments, however, addressed the accuracy of the perturbation scheme in the presence of a long large underlying wave. We conducted a laboratory experiment in which both a long wave and resonantly interacting intermediate-length waves were generated and we measured the growing short wave. 
Our experiment provides a basis of comparison for our numerical findings on the influence of the long wave on the nonlinear interactions amongst the intermediatelength waves.

A principal finding here is that a long wave significantly alters energy transfer to a short wave from intermediate-length waves with prescribed linear amplitude components. In addition to the numerical and experimental data, we are able to provide a physical explanation for why this occurs.

The results have implication for computations of nonlinear-energy transfer in a wave spectrum. This is complicated by the fact that the high-frequency tail of a measured wave spectrum is influenced not only by the usual linear wave spectrum proportional to the squares of wave amplitudes, but also significantly by the quadratic spectrum which depends on the fourth power of wave amplitude. In an appendix we derive expressions for the quadratic spectrum for unidirectional seas. Computations of nonlinear energy transfer in a wave spectrum require the full directional spectrum, but the simpler unidirectional spectrum is sufficient for showing that use of measured spectral values for computing nonlinear energy transfer to short waves must be done with care and caution.

\section{The nonlinear wave-wave interactions}

It is now well known that wave components exchange energy through nonlinear interactions. For gravity waves, the resonant nonlinear interaction generally requires a quartet of waves. The resonance conditions for the frequencies and wavenumbers under which these interactions take place can be written as (see Phillips 1960)

$$
\left.\begin{array}{l}
\omega_{1} \mp \omega_{2} \mp \omega_{3} \mp \omega_{4}=0, \\
\boldsymbol{k}_{1} \mp \boldsymbol{k}_{2} \mp \boldsymbol{k}_{3} \mp \boldsymbol{k}_{4}=0,
\end{array}\right\}
$$

provided that the linear dispersion relation holds for each wave component:

$$
\omega_{n}^{2}=g k_{n} \text { for } n=1,2,3,4,
$$

where $g$ is the acceleration due to gravity. When the resonance conditions are satisfied, the third-order nonlinear interactions between three waves, which we call primary waves or primaries, result in a forcing function that has exactly the same frequency and wavenumber as the fourth. Since the fourth wave is a free wave, by virtue of its frequency and wavenumber satisfying the dispersion relation, it grows when forced at its own frequency and wavenumber, thereby obtaining energy from the other waves. The growing wave is called the tertiary wave. When the first three interacting waves, but not the fourth, are initially present, the amplitude of the tertiary wave, $a_{t e r}(t)$, behaves initially as

$$
\left|a_{t e r}(t)\right|=K t,
$$

where $K$ is the interaction (coupling) coefficient involving the wavenumbers, wave frequencies and the first-order amplitudes of the first three wave components.

Of all possible wavenumber configurations of the vectors $k_{1}, k_{2}, k_{3}$, and $k_{4}$, one is particularly convenient for theoretical and numerical studies. As illustrated in figure $1(a)$, two of the primary wave trains coincide (say $k_{1}$ ) and are perpendicular to another $\left(k_{2}\right)$ so that there are only three distinct wavenumbers in the interacting resonant quartet and the wavenumber of the resonant tertiary wave is given by

$$
\boldsymbol{k}_{t e r}=2 \boldsymbol{k}_{1}-\boldsymbol{k}_{2} \text {. }
$$


The resonance conditions for this special triad require that the following equation along with (4) be also satisfied:

$$
\omega_{t e r}=2 \omega_{1}-\omega_{2}
$$

where the linear dispersion relation holds for each component. Combining the resonance conditions with the linear dispersion relation determines the theoretical ratio for the primary wave frequencies $\omega_{1} / \omega_{2}=1.73567$ (Longuet-Higgins \& Smith 1966). From the ratio of the primary wavenumbers, the tertiary wave is found to make an angle of $9.42^{\circ}$ with $\boldsymbol{k}_{1}$. A prescribed wave frequency for one of the primaries defines the characteristics of the other members of the triad.

Phillips (1960) and later Longuet-Higgins (1962) studied this special triad utilizing perturbation theory and they derived theoretical predictions having the form of (3) for the initial growth of the resonant tertiary wave. This special case was also used as a bench-mark in the aforementioned experiments. In the theory of Longuet-Higgins (1962), the fact that the tertiary wave grows at the expense of the primary waves is not accounted for and the amplitudes of the primary wave components are treated as constants throughout the course of the interaction. For the set of waves in the canonical situation considered here, the initial growth rate does not continue since the tertiary wave interacts with the others once it has grown to finite size. Thus the physical interpretation of (3) should be restricted to a brief initial time which is small in comparison with the interaction time. This limitation was overcome by Benney (1962) who showed that the energy sharing mechanism in the interacting quartet by analysing the full problem of energy exchanges amongst a set of four resonant waves satisfying (1). Based on the original work of Zakharov (1968) and the subsequent work of Crawford, Saffman \& Yuen (1980), Stiassnie \& Shemer (1984) derived a set of coupled nonlinear equations governing the evolution of the amplitude of each discrete mode involved in the quartet interaction up to third order. We shall see that to make the theory based on perturbations about the mean free surface accurate for the case at hand with the long wave, higher-order contributions from the spectral method are required.

In view of the wealth of background information on the interacting special triad, we used it as a 'base case' for our numerical and experimental investigations. Then we added the underlying long wave having wavenumber $\boldsymbol{k}_{3}$ such that the wavenumber diagram took the form of figure $1(b)$. Numerical and experimental studies were conducted on this set of waves. The important point here is that the long-wave amplitude is not necessarily small in comparison to the tertiary wavelength. By taking this approach we were able to assess the accuracy of the theoretical and numerical methods in addition to our principal objective which was determination of the influence of the long wave on the resonant interaction between the other waves. We found that the presence of the long wave modifies the growth of the tertiary wave, and why this happens.

\section{Numerical methods}

A direct numerical method that avoids the perturbation theory approximation was developed by Ölmez (1991) to compute the energy transfer for a quartet of waves. A spectral method program was also developed by us for further comparisons with the direct numerical method. The spectral method is a numerical application of perturbation theory, achieving numerical efficiency by using Fourier expansions in the horizontal domain, which includes perturbations of arbitrarily high order, thereby avoiding the third-order limitation of the conventionally used methods. 
For both methods, the approach taken is to consider flows governed by a velocity potential, $\phi$, satisfying Laplace's equation beneath a time varying free surface of elevation, $\zeta$. The time evolution is determined by stepwise integrating the kinematic and dynamic free-surface boundary conditions:

$$
\begin{gathered}
\frac{\partial \zeta}{\partial t}=w(1+\nabla \zeta \cdot \nabla \zeta)-\nabla \phi_{S} \cdot \nabla \zeta \quad \text { on } \quad z=\zeta, \\
\frac{\partial \phi_{S}}{\partial t}=-g \zeta-\frac{1}{2} \nabla \phi_{S} \cdot \nabla \phi_{S}+\frac{1}{2} w^{2}(1+\nabla \zeta \cdot \nabla \zeta) \quad \text { on } z=\zeta,
\end{gathered}
$$

where $\phi_{S}$ and $w$ represent the velocity potential and the vertical velocity on the free surface. In the above equations, $\boldsymbol{\nabla}=(\partial / \partial x, \partial / \partial y)$ denotes the horizontal gradient. The time integration provides updated values of the surface elevation and velocity potential. In order to completely specify the flow so that a subsequent time-step integration can be carried out, the vertical velocity, $w$, must be found. The difference between the direct and the spectral methods lies in the way $w$ is determined.

For our calculations with the spectral method, the time integration of the freesurface boundary conditions is carried out using a fourth-order explicit Runge-Kutta method with a constant time step. For the direct method, a fourth-order multi-step Adams-Bashforth-Moulton method is preferred over Runge-Kutta owing to its computational efficiency since the numerical technique is computationally expensive. Various time-step sizes were tested in each of our calculations. A general finding was that a good time-step size is 2 to $3 \%$ of the shortest fundamental wave period in the problem under consideration. No significant gains in accuracy were achieved with shorter time steps.

During the time-stepping procedure, a high-wavenumber instability on the free surface develops which, if not suppressed, eventually causes the numerical scheme to break down. This type of instability, often referred to as sawtooth, has been reported by several investigators (Longuet-Higgins \& Cokelet 1976; Baker, Meiron \& Orszag 1982; Dommermuth et al. 1988). We adopted a fast Fourier transform (FFT) technique by which all the high-wavenumber instabilities are filtered out. The surface wave elevation and the velocity potential are transformed into the Fourier space by a two-dimensional FFT, and all the higher-order harmonics that are above the wavenumber at which the instability is detected are filtered out. Then transforming back into the physical space by an inverse FFT, the computations are carried out for subsequent time steps.

\subsection{Direct numerical evaluation}

In our direct numerical procedure, the normal derivative of the potential is determined by solving the Green's theorem integral equation:

$$
-2 \pi \phi(p)=f f \phi(q) \frac{\partial G(p, q)}{\partial n(\boldsymbol{q})} \mathrm{d} S(\boldsymbol{q})-\iint G(\boldsymbol{p}, \boldsymbol{q}) \frac{\partial \phi(\boldsymbol{q})}{\partial n(\boldsymbol{q})} \mathrm{d} S(\boldsymbol{q}),
$$

where the first integral is in the sense of a Cauchy principal value and excludes integration over the field point $\boldsymbol{p}$. The singularity is located at the source point $\boldsymbol{q}$. $G(p, q)=1 /|\boldsymbol{r}|$ is the three-dimensional free-space Green function where $r=|\boldsymbol{p}-\boldsymbol{q}|$ is the distance between the source and field points.

The solution is carried out over the surface, $S$, of a domain defined by the calculated free-surface shape at each time and vertical sidewalls that intersect in a rectangular prism. With the resulting knowledge of the normal derivative of the potential, the vertical component can be calculated since the surface normal vector and values of the 
potential on the surface are known. By alternately time stepping (6) and (7) and solving the integral equation (8), the surface elevation and the velocity potential as functions of time are obtained. After each time step, the two-dimensional Fourier transform of the free-surface shape is calculated to separate and evaluate the amplitude of the tertiary wave.

Since deep-water waves are considered, the computational domain can be freed of the bottom face provided that the domain is deep enough for there to be negligible flow at its bottom. Spatially periodic solutions are considered so that the boundary conditions on the side faces become periodicity conditions. We solve the integral equation (8) numerically by discretizing the boundary $S$ into $N$ quadrilateral panels, and by satisfying the equation at a prescribed collocation point on each panel. Collocation points are chosen at the centroid of each panel where the boundary conditions are invoked. The simplest form is to consider the singularity strengths to be constant on each panel and each panel to lie in a plane. Each integral in (8) now depends only on the form of the Rankine source Green function, $G$, and the geometry of the panels. Explicit expressions for these integrals, which we have used in our numerical implementation, are given by Newman (1986). This direct numerical evaluation is also referred to as the boundary integral equation method (BIEM). Specific details of our BIEM are given in Ölmez (1991) and in Ölmez \& Milgram (1995).

\subsection{Evaluation by the spectral method}

Dommermuth \& Yue (1987) and West et al. (1987) developed high-order spectral methods for the study of nonlinear gravity waves. These references provide complete descriptions so we only summarize the method here.

The spectral method also uses (6) and (7) to march the surface potential and elevation forward in time. It differs from the direct numerical method in the way it evaluates the vertical derivative of the potential after each time step.

The spectral method presupposes that the velocity potential can be expanded as a regular perturbation expansion in the following form:

$$
\Phi(x, z, t)=\sum_{m=1}^{N} \Phi_{m}(x, z, t)
$$

where $x=(x, y)$ and $M$ denotes the order of expansion adopted in the procedure. Following Dommermuth \& Yue (1987), each $\Phi_{m}$ is further expanded in a Taylor series about $z=0$ and the surface potential is obtained in the following form:

$$
\Phi_{S}(x, t)=\Phi(x, \zeta, t)=\sum_{m=1}^{M} \sum_{k=0}^{M-m} \frac{\zeta^{k}}{k !} \frac{\partial^{k}}{\partial z^{k}} \Phi_{m}(x, 0, t) \text {. }
$$

Expanding (10) and collecting terms at each order, provides the following sequence of equations for the unknown $\Phi_{m}$ in terms of the surface potential $\Phi_{S}(x, t)$ :

$$
\begin{gathered}
\Phi_{1}(x, 0, t)=\Phi_{S}(x, t), \\
\Phi_{m}(x, 0, t)=-\sum_{k=1}^{m-1} \frac{\zeta^{k}}{k !} \frac{\partial^{k}}{\partial z^{k}} \Phi_{m-k}(x, 0, t), \quad m=2, \ldots, M .
\end{gathered}
$$

The velocity potential at each order $m$ (assuming periodic boundary conditions), for a wave field composed of $N$ free wave modes, is expressed by the following sum:

$$
\Phi_{m}(\boldsymbol{x}, z, t)=\sum_{n=1}^{N} \phi_{m, n}(t) \mathrm{e}^{\left|\boldsymbol{k}_{n}\right| z} \mathrm{e}^{\mathrm{i} \boldsymbol{k}_{n} \cdot \boldsymbol{x}}, \quad m=1,2, \ldots, M,
$$


where the $\boldsymbol{k}_{n}$ are the wavenumber harmonics of the horizontal domain, $\boldsymbol{k}=\left(k_{x}, k_{y}\right)$, and deep water is implied. For each time, the modal amplitudes, $\phi_{m, n}$, are found by substituting (13) into (11) and (12), and solving for the unknowns as a function of lower orders. The vertical velocity is then approximated by the following expression:

$$
w=\sum_{m=1}^{M} \sum_{k=0}^{M-m} \frac{\zeta^{k}}{k !}\left[\frac{\partial^{k+1}}{\partial z^{k+1}} \sum_{n=1}^{N} \phi_{m, n}(t) \mathrm{e}^{\left|k_{n}\right| z} \mathrm{e}^{\mathrm{i} k_{n} \cdot x}\right]_{z=\mathbf{0}} .
$$

Fast Fourier transforms are employed for moving back and forth between the physical and Fourier domains. For the time-stepping procedure, all the horizontal gradients of the surface potential and the wave height are performed in the Fourier domain. Starting with prescribed initial conditions for $\phi_{S}\left(x, t_{0}\right)$ and $\zeta\left(x, t_{0}\right)$, the freesurface boundary conditions are integrated in time over equally spaced collocation points, and the new values of the surface potential and the free-surface shape are computed in the physical domain. Based on the above approach, we prepared a computer algorithm for solving the nonlinear wave problems presented herein.

\section{Experimental apparatus and procedure}

The experiments were carried out in a rectangular tank at the Institute for Marine Dynamics (IMD) of the National Research Council of Canada. The overall horizontal dimensions of the IMD tank are $75 \mathrm{~m}$ by $32 \mathrm{~m}$ and the maximum depth is $3.5 \mathrm{~m}$. There are wavemakers on two adjacent sides of the tank with wave absorbers on the two remaining sides.

The wavemakers are segmented, with each segment hydraulically driven by a feedback control system so as to follow an applied electrical input signal. Each segment can have an arbitrary amount of piston-like or rotating flap-like motion. We operated the segments using piston-mode. All the segments on a single side were given identical motions to create intersecting (nearly) two-dimensional wave trains. Small amounts of leakage between adjacent segments result in small deviations from two-dimensionality. At the corner of the tank where the wavemakers would intersect, they are replaced by a solid rectangle which projects into the tank beyond the wavemakers by $a=0.185 \mathrm{~m}$ on one side and $b=0.675 \mathrm{~m}$ on the other side as shown in figure 2 .

In order to test the linearity of the wavemaker system, waves from sinusoidal input signals in the frequency and amplitude ranges planned for the experiment were made by the wavemakers along one wall with those on the adjacent wall stationary. These waves were Fourier-analysed to assess their harmonic content. The ratios of the first and second harmonic amplitudes to that of the fundamental generally agreed with Stokes wave theory to within $1 \%$ of the fundamental amplitude, although occasional errors up to $3 \%$ were observed. These errors correspond to 0.3 and $0.9 \mathrm{~mm}$ respectively. In all likelihood, they are due to variations in the shapes of the menisci of the water against the wave gauge wires which vary according to surface contamination. This was minimized by frequent cleaning.

Wave absorbers at the IMD facility are installed on the two tank sides opposite the wavemakers and are of the variable-porosity type described by Jamieson \& Mansard (1987). Information from earlier tests done at IMD gives wave reflection coefficients of about $3 \%$ for the fundamental amplitudes and frequencies of our experiments. However, our data on the spatial variation of wave amplitudes indicate that the reflection coefficient could be as large as $10 \%$. The dominant resonant group involving reflected waves contains the original $k_{1}$ component and the reflected $k_{2}$ component. To 


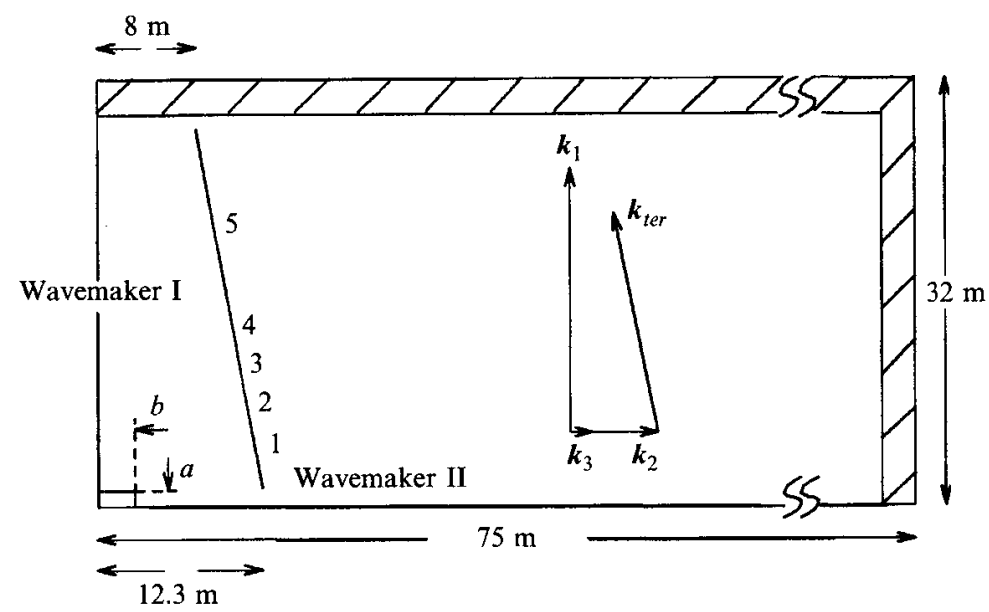

FIGURE 2. Plan view of the wave tank at IMD.

$\begin{array}{ccccc} & \text { Wavenumber } & \text { Frequency } & \text { Wavelength } & \text { Period } \\ \text { Wave } & |\boldsymbol{k}| /\left|\boldsymbol{k}_{\mathrm{2}}\right| & \omega / \omega_{2} & \lambda / \lambda_{2} & T / T_{2} \\ 1 & 3.01255 & 1.73567 & 0.33194 & 0.57615 \\ 2 & 1.00000 & 1.00000 & 1.00000 & 1.00000 \\ \text { Tertiary } & 6.10753 & 2.47134 & 0.16373 & 0.40464\end{array}$

TABLE 1. Data for the special triad

avoid an error from this effect, in our experiments the $\boldsymbol{k}_{2}$ wave propagated in the long tank direction and we used the data acquired before the reflected $\boldsymbol{k}_{2}$ wave reached the wave gauges (see figure 2) in most cases (see below).

Measurements of the free-surface elevation were made by an array of resistance-type wave gauges made of two parallel 30 -gauge copper wires spaced $1 \mathrm{~cm}$ apart. These wave gauges were calibrated by lowering and raising the wires through a known distance in calm water as well as by measuring sinusoidal waves with independently determined amplitudes. Five wave gauges were located at distances of 4.0, 6.0, 8.0, 10.0 and $19.7 \mathrm{~m}$ away from the wavemaker in the direction of the tertiary wave propagation over which the interaction takes place. According to the theory in $\$ 2$, the tertiary wave at resonance is at an angle of $9.42^{\circ}$ to the direction of the first primary wave (away from the second wavemaker with a slight component towards the first wavemaker). This generates a narrow reflection region from the first wavemaker. Hence the last wave gauge was located $9.07 \mathrm{~m}$ away from the first wavemaker to keep it out of the reflection zone. A plan view of the tank along with the gauge locations is shown in figure 2.

Measurements of the outputs from the five wave gauges were made with a 12-bit Ato-D converter and recorded on a computer in real time for subsequent data analysis. Each run consisted of 4096 points per gauge channel taken at a sampling frequency of $16 \mathrm{~Hz}$ which corresponds to $256 \mathrm{~s}$ of data, beginning $30 \mathrm{~s}$ after the wavemakers were turned on. At each sample time, all five channels were sampled in a time period of $0.04 \mathrm{~ms}(0.01 \mathrm{~ms}$ delay between channels). The $16 \mathrm{~Hz}$ sampling rate was chosen to avoid aliasing based on initial studies of measured data showing that spectral levels above $5 \mathrm{~Hz}$ were less than $0.5 \%$ of the mean levels of the primary $\left(k_{1}\right.$ and $\left.k_{2}\right)$ waves. To maximize the dynamic range and the signal-to-noise ratio of the high-frequency 
tertiary wave data, the wave gauge system frequency response increased with frequency in the data range in a manner similar to that used by Ölmez \& Milgram (1992).

The wavemakers were operated with primary wave circular frequencies $\omega_{1}=6.974 \mathrm{rad} \mathrm{s}^{-1}$ and $\omega_{2}=4.018 \mathrm{rad} \mathrm{s}^{-1}$ and as such the ratio $\omega_{1} / \omega_{2}$ yields the theoretical value required for the resonant interaction. The long primary and swell (long wave) were generated simultaneously and propagated along the long side of the tank. The short primary wave propagated long the short side of the tank.

Spectral analysis was used to analyse the frequency distribution of wave energy at each wave gauge. The energy distribution in the wave field can be deduced from its power spectrum defined as

$$
S(\omega)=\frac{2}{T}\left|\int_{0}^{T} z(t) \mathrm{e}^{\mathrm{i} \omega t} \mathrm{~d} t\right|^{2},
$$

where $T$ is the length of the portion of the record used and $z(t)$ is the data record from the measurements. The initial factor of 2 makes the spectrum one-sided. Tertiary wave amplitudes were computed at each gauge location from the integral of the surface elevation spectrum around the fundamental tertiary wave frequency:

$$
a\left(\omega_{t e r}\right)=\left[2 \int_{\omega_{t e r}-\Delta \omega}^{\omega_{t e r}+\Delta \omega} S(\omega) \mathrm{d} \omega\right]^{1 / 2},
$$

where $\Delta \omega$ was chosen to be $\omega_{t e r} / 50$.

For a few runs, the entire $256 \mathrm{~s}$ record was divided into eight records, each $32 \mathrm{~s}$ long. These were individually analysed to determine the standard deviation of the measured tertiary wave amplitude. For the $32 \mathrm{~s}$ records, the standard deviation was $19.5 \%$ of the tertiary amplitude.

Starting when the wavemakers are turned on, the tertiary wave reaches full theoretical growth at all wave gauges after $39 \mathrm{~s}$ and the reflection of the long primary wave from the tank wall absorber first reaches a wave gauge in $113 \mathrm{~s}$. Therefore, the portion of the data shown in figures $4(a), 6(b)$ and 7 used in studying tertiary wave evolution was from 39 to $103 \mathrm{~s}$ after the wavemakers were turned on, corresponding to 9 to $73 \mathrm{~s}$ in the data records. This provided data sections of 1024 points for which the standard deviation of measured tertiary wave amplitudes is $13.8 \%$. For the cases shown in figures $4(b)$ and $6(a)$, wave disturbances, probably related to wavemaker startup, evident in the early portions of the data record led us to analyse 1024 point data segments starting after the $k_{2}$ reflected wave reached the wave gauges.

\section{Numerical and experimental results}

We first consider the special triad alone and compute the growth of the resonant tertiary wave for primary wave steepnesses, $\epsilon$, of 0.1 and 0.2 . The smaller steepness is used for benchmark purposes. Then, moderately steep waves are considered for testing the accuracy of the perturbation analyses. These numerical results are compared with our experimental measurements for the special triad. Then, a long wave whose amplitude is of the order of the tertiary wavelength is added to the special triad and the tertiary wave growth is computed under the influence of the long wave. These computations are also compared with the experimental results to confirm the validity of our numerical findings.

The tertiary wave amplitudes in the experiments are very small, typically a few millimetres. Although fractional differences between numerical results and experiment are sometimes substantial, in almost all instances the actual amplitude difference is less 
than $1 \mathrm{~mm}$. Of the 22 regularly analysed data points shown subsequently, 16 of them show differences in amplitude between experiment and computation of less than $1 \mathrm{~mm}$. The remaining six points have differences between experiment and computation of less than $2 \mathrm{~mm}$.

\subsection{Results for the special triad alone}

The special triad we will consider is composed of two primary waves $\left(\boldsymbol{k}_{1}\right.$ and $\left.\boldsymbol{k}_{2}\right)$ and the resonant tertiary wave as shown in figure 1 . Based on the theoretical ratio of wave frequencies for resonance, table 1 summarizes the characteristics of each member of the triad.

The numerical solution to the boundary-value problem requires that the free-surface shape and the velocity potential be prescribed at the initial instant of time. We found that the numerical calculations are sensitive to initial conditions in that errors in them introduce standing waves in the system. We used perturbation theory to calculate initial conditions for the interacting triad up to second and third order in wave slope (Ölmez 1991), with the latter naturally introducing fewer of the erroneous standing waves. Using these, the subsequent computations with the BIEM were fully nonlinear whereas the spectral method computations were carried out to the fifth order.

For the BIEM, the free-surface geometry is approximated by 32 elements in each direction. Along the depth, which is half the wavelength of the longer primary, 20 elements were used with a quarter-period cosine-spacing distribution, dense at the top. Filtering is applied at every time step such that wavenumbers above the eighth harmonic of the fundamentals are filtered out. With tenth-order filtering, a sawtooth appearance on the surface took over soon after one wave period of the long primary, at which point the departure from the initial energy and momentum was considerable. This does not occur with the eighth-order filtering.

For the spectral method, 64 elements are used in each direction on the free surface. This provides 32 unaliased modes in each direction. With this spatial resolution, convergent results are obtained with a fifth-order $(M=5)$ approximation. Despite having fifth-order accuracy, Fourier modes up to the tenth harmonic of the fundamentals are preserved in the computations. Therefore, smoothing is applied at every time step such that wavenumbers higher than the tenth harmonic of the fundamentals are eliminated. A time step of $T_{1} / 50$ is used for both methods. Convergence with time-step size is examined in Ölmez (1991) and $\Delta t=T_{1} / 50$ is found to be quite satisfactory.

Figures $3(a)$ and $3(b)$ show our computed tertiary wave amplitudes for primary wave steepnesses of 0.1 and 0.2 with second- and with third-order initial conditions, respectively. The normalized amplitude, $\left(a_{t e r}\right)_{N}$, shown on the ordinates of the figures is

$$
\left(a_{t e r}\right)_{N}=\frac{a_{t e r} k_{t e r}}{\left(a_{1} k_{1}\right)^{2}\left(a_{2} k_{2}\right)} .
$$

The theoretical results shown in the figures are based on (3) as given by the classical theory (Longuet-Higgins 1962), and are normalized according to (17):

$$
\left(a_{t e r}\right)_{N}=2.77968 \mathrm{D} / \lambda_{t e r},
$$

where $D$ is the distance over which the interaction takes place.

Computations with our BIEM were performed only up to $D=3 \lambda_{\text {ter }}$ because of the computationally expensive nature of this numerical scheme. Note that the numerical computations display regular oscillations in the amplitude of the tertiary wave when the second-order initial conditions are used. These oscillations were much smaller when 
(a)

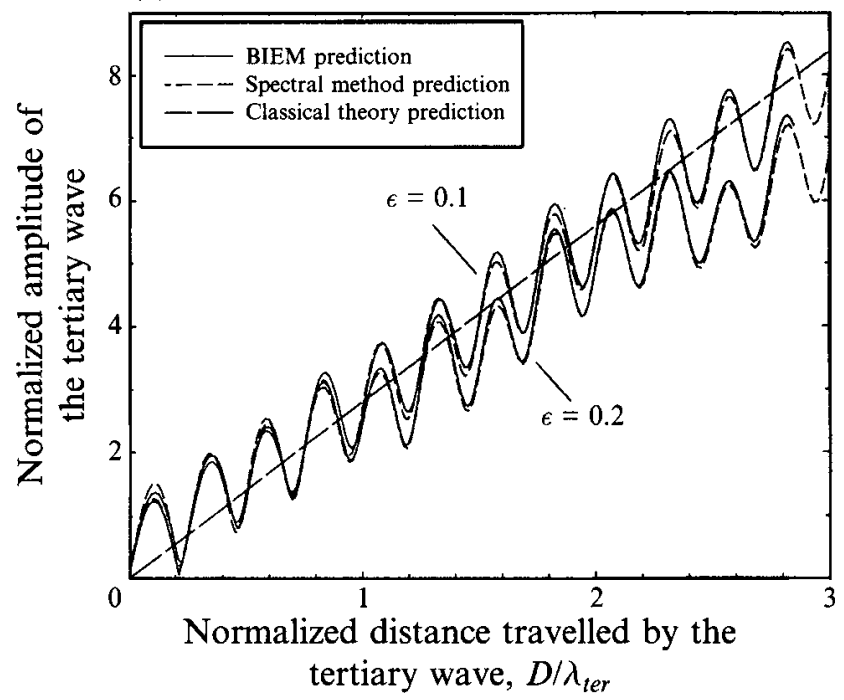

(b)

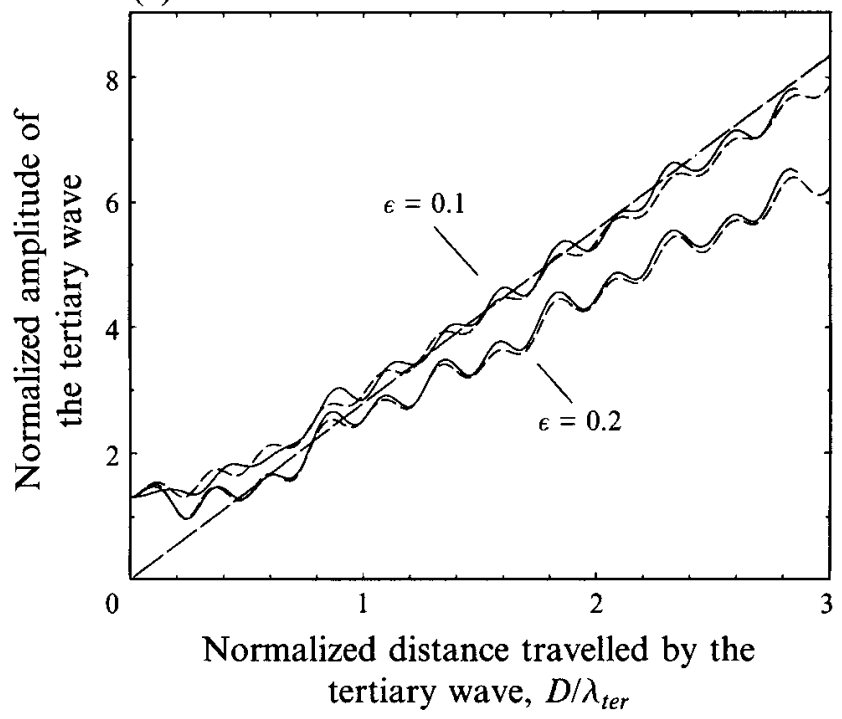

FIGURE 3. Growth of the tertiary wave for $(a)$ second-order initial conditions, and $(b)$ third-order initial conditions.

initial conditions were accurate to the third order. An analysis of the relative phases between the first-order velocity potential and the wave elevation shows that these oscillations are consistent with their being due to standing waves. It is important to note that the oscillations are essentially identical for the entirely separate direct and spectral methods lending weight to the conclusion that they are associated with the initial conditions rather than numerical errors. For the nonlinear computations to be exact, purely travelling nonlinear waves are required for the initial conditions whereas we have used conditions for travelling waves up to third order.

For the second-order initial conditions, the tertiary wave starts with a zero amplitude whereas for the third-order initial conditions, the tertiary wave has a nonzero amplitude at $t=0$. This is a steady-state, bound component which is in 
quadrature with the growing component and whose existence is due to crossinteractions of the primary waves at the third-order as quantified by Ölmez (1991). The growing tertiary wave component has a zero initial amplitude.

From figures $3(a)$ and $3(b)$, a comparison between the spectral method and the BIEM reveals that both methods are in remarkably good agreement. As a check on the global numerical accuracy of the BIEM, total energy and horizontal momenta are computed at every time step. These quantities are well conserved (to within $0.05 \%$ and $0.1 \%$ for the lower and higher steepnesses, respectively) during the entire simulation. For mild nonlinearities, the accuracy of the classical perturbation theory is good for the initial growth of the tertiary wave. For steeper primary waves, the accuracy of the perturbation approximation is restricted to a briefer initial time, which is consistent with the analysis of Tomita (1989).

To provide a basis for the accuracy of the low-order mode-coupled equations in the long-term evolution of the wave field, we consider the results from the low-order modecoupled equations (Stiassnie \& Shemer 1984) and compare them with those from the high-order spectral method and from the experiment.

The modal amplitudes in the triad for the mode-coupled equations are obtained by solving a system of three nonlinear complex ordinary differential equations (Stiassnie \& Shemer 1984, equation (4.1)) in time. A fourth-order Runge-Kutta method is employed with a time step of $T_{1} / 120$. The typical interaction distance needed for the tertiary wave to be comparable to a primary wave in size is predicted to be (Phillips 1960):

$$
D_{i n t}=\frac{1}{2} \frac{\lambda_{t e r}}{\left(a_{1} k_{1}\right)\left(a_{2} k_{2}\right)} .
$$

Figures $4(a)$ and $4(b)$ show the long-term interaction of the special triad for two steepnesses. For both primary waves having a steepness of 0.1 , the typical interaction distance requires $50 \lambda_{t e r}$. From figure $4(a)$, both the spectral method and the modecoupled equations predict the first maximum of the tertiary wave to occur near $48 \lambda_{\text {ter }}$ which closely corresponds to the theoretical interaction distance found above. Aside from a slight discrepancy in the maximum amplitude of the tertiary wave, modecoupled equations performed well at this steepness. The higher-steepness case has $a_{1} k_{1}=0.174$ and $a_{2} k_{2}=0.180$, which are the steepnesses achieved in the experiment. For this case, the theoretical interaction distance reduces to $16 \lambda_{\text {ter }}$ because of stronger nonlinearity. The first maximum of the tertiary wave is predicted near $16.5 \lambda_{t e r}$ and $17 \lambda_{t e r}$ by the mode-coupled equations and the spectral method, respectively. Although the mode-coupled equations do not completely agree with the spectral method for the entire simulation, the overall behaviours are similar. These comparisons validate the accuracy of mode-coupled equations for small steepnesses, with discrepancies being apparent at the higher steepnesses. Our experimentally observed amplitudes of the tertiary wave at the five wave gauge locations are also plotted in figures $4(a)$ and $4(b)$. Agreement between experiment and the numerical computation is quite good except for the last data point in each case which appears to be in error by about $1.9 \mathrm{~mm}$ in amplitude.

\subsection{Results for the special triad on a long underlying wave}

When a long wave whose amplitude is comparable to the tertiary wavelength is added to the wave field, the displacement from the equilibrium level for the tertiary wave will be on the order of its wavelength. This clearly violates the size restriction on the small expansion parameter of a perturbation expansion about the mean free surface for the short wave of interest. It would be possible to develop an expansion about the surface 
(a)

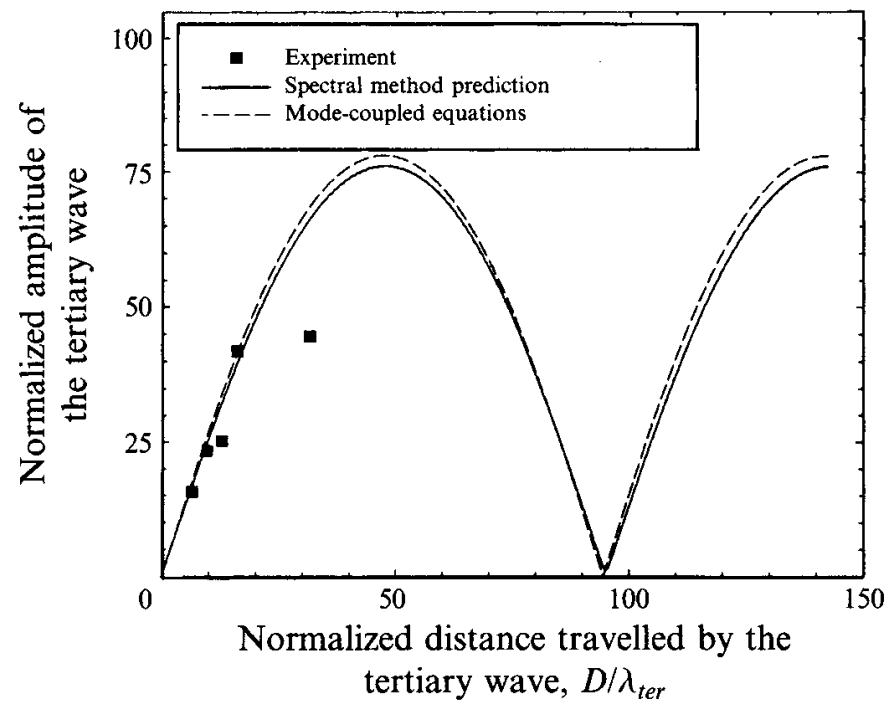

(b)

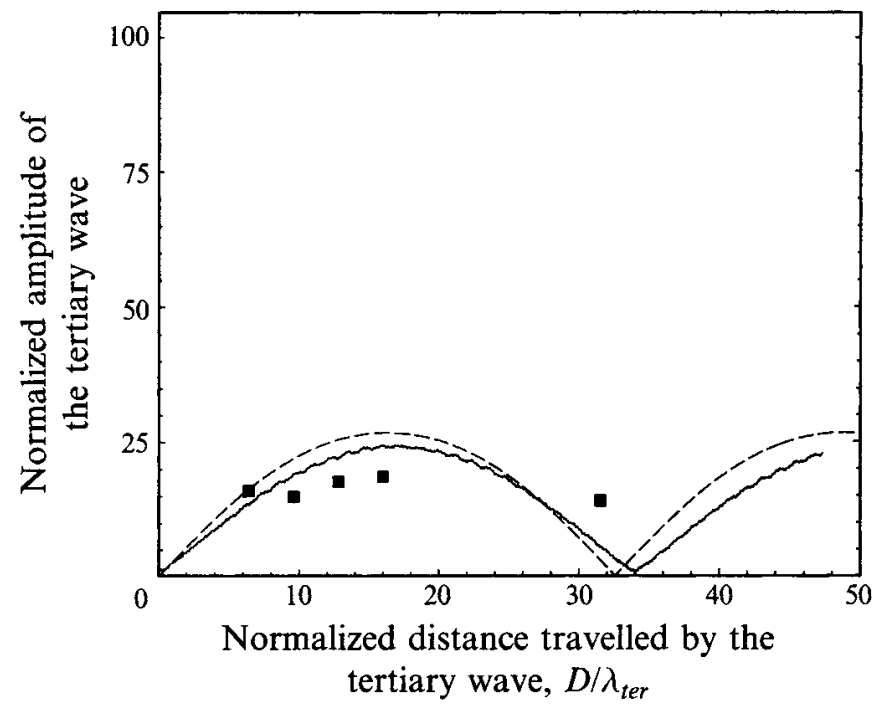

Figure 4. A comparison of the mode-coupled equations with the spectral method and the experiment for primary wave steepness of $(a) 0.1,(b) 0.174$ and 0.180 .

of the long wave to avoid this violation. On the other hand, we shall find that the same spectral method used above is accurate for the case at hand, in spite of the larger expansion distances.

The first objective here is to compute the initial tertiary wave growth under the influence of the long wave and make a comparison between results of the direct numerical method which has no size restriction and those methods that rely on perturbation analysis. Once we establish the validity of the more computationally efficient spectral method calculations, these will be used in predicting the long-term evolution of the tertiary wave for further comparisons with the experiments.

The long wave added to the special triad is chosen to be propagating in the same 


$\begin{array}{ccccc}\text { Wave } & \text { Wavenumber } & \text { Frequency } & \text { Wavelength } & \text { Period } \\ 3 & |\boldsymbol{k}| /\left|\boldsymbol{k}_{2}\right| & \omega / \omega_{2} & \lambda / \lambda_{2} & T / T_{2} \\ & 0.25 & 0.50 & 4.00 & 2.00\end{array}$

TABLE 2. Characteristics of the long-wave component

direction as $k_{2}$ with a wavelength of $4 \lambda_{2}$. Table 2 gives the characteristics of the longwave component. The width of the computational domain in the $y$-direction is equal to the long wavelength along which we used 64 panels. This leaves 16 panels per wavelength for the longer primary wave which also travels in the same direction. The length of the domain in the $x$-direction is equal to the wavelength of the short primary wave, $\lambda_{1}$. The presence of the long wave makes this computational task quite a demanding one for the BIEM. Therefore, we used only 16 panels in the $x$-direction, maintaining the same spatial resolution for the primary waves. As before, 20 panels are used along the depth, which is now chosen to be half the wavelength of the long wave. Filtering is applied at every time step using fifth-and 17th-order filtering in the $x$-and $y$-directions, respectively. With our current spatial resolution, filtering at the above levels was needed to keep the simulation free of high-wavenumber instabilities. For the spectral method, we set the maximum order in the perturbation expansion to $M=5$ and used 16 and 64 unaliased modes in the $x$ - and $y$-directions, respectively, for the same computational domain. Filtering for the spectral method is also applied at every time step by removing the Fourier modes higher than the sixth- and 21st-harmonics of the fundamentals in the $x$ - and $y$-directions. As the BIEM is computationally expensive, we could not maintain the same spatial resolution in the BIEM as in the spectral method. This led to different filtering parameters in each approach. For both methods, $\Delta t=T_{1} / 50$ is used as the time-step size.

The initial conditions for this case are calculated by a third-order perturbation analysis. The presence of a fourth wave component makes the analysis 'by hand' onerously complicated. Therefore, the analysis for initial conditions was worked out to third-order using MACSYMA, a program that can handle algebraic manipulations symbolically. Using MACSYMA, we also confirmed the results of our third-order 'hand calculations' for the initial conditions of the special triad alone.

Figure 5 shows computed results and those from the classical theory which bases the growth rate on the initial amplitudes of the linear components. The steepnesses for the primary waves were chosen to be 0.1 . The computations plotted in figure 5 were carried out for swell steepnesses of 0.05 and 0.1 . The most important result is that the numerical methods show a large reduction in the growth of the tertiary wave when the long wave underlies the special triad. This is shown by both the BIEM and the spectral method. These two numerical methods show some local differences, but are in good global agreement.

Considering the relatively few control points used on the free surface for the run with the BIEM, it is essential to confirm the convergence with the spatial resolution. We could not double the number of control points on the surface as it was not computationally feasible. However, we were able to use twice as many panels in the $x$ direction and compute the tertiary wave amplitude for swell steepness of 0.1 as shown by the dotted lines. It follows closely the one with fewer panels. We found this convincing for the convergence, and did not perform the entire simulation.

In addition to the effects of small standing waves due to limiting initial conditions to third-order, figure 5 shows a periodicity in the growth rate with a period of 1.2 of 


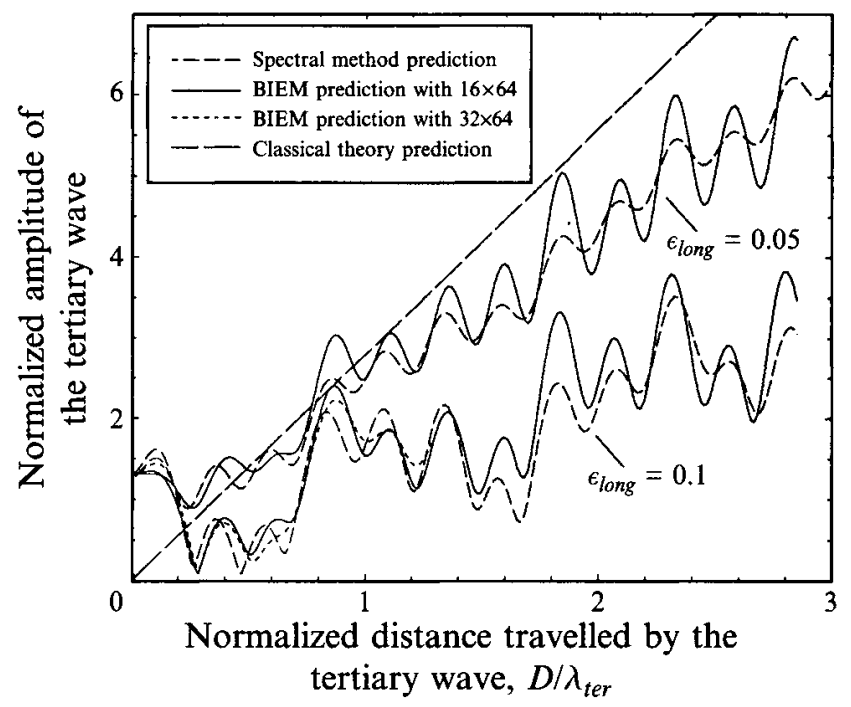

FIGURE 5. A comparison of the spectral method with the BIEM in the presence of an underlying long wave with steepnesses of 0.05 and 0.1 .

the normalized units of the abscissa of the figure. This periodicity is also particularly evident in the later figure 7. An analysis of the numerical system has shown that this is due to the time-varying phase differences of the long wave and the $\boldsymbol{k}_{2}$ primary wave which propagate in the same direction. The computational domain length contains one long wavelength and four lengths of the $\boldsymbol{k}_{2}$ wave. As time progresses, the long wave overtakes the $k_{2}$ wave by virtue of its larger phase velocity. This leads to a periodic time structure with period equal to $\lambda_{2}$ divided by the difference in the phase velocities of the long and $k_{2}$ waves. This period is $1.56 \mathrm{~s}$ which corresponds to normalized distances of 1.23 units in the figures. The space-time transformation for these figures is based on the group velocity of the tertiary wave.

In view of the good agreement between the BIEM and the spectral method in the presence of the long underlying wave, we will use the spectral method to predict the long-term evolution of the tertiary wave for comparisons with experiment. Figure 6(a) and $6(b)$ show the tertiary wave amplitudes from the experiment and the spectral method. These figures also contain spectral method results for tertiary wave growth in the absence of the long wave to help demonstrate the effect of the long wave on tertiary wave growth.

In figure $6(a)$, the primary waves have a steepness of 0.1 and co-exist with a long underlying wave of steepness 0.05 . The measured amplitudes at the three middle points are in good agreement with the results of the spectral method. The first point differs by an amount corresponding to $1.4 \mathrm{~mm}$ and the last point by $1.8 \mathrm{~mm}$.

In the next run, whose results are in figure $6(b)$, we increased the steepness of the first and second primary waves to 0.174 and 0.180 respectively. The swell amplitude was kept the same. In this case, the numerical computation broke down after a time of $13.9 \mathrm{~s}$ which corresponds to a propagation distance of 11 tertiary wavelengths on the basis of the tertiary wave group velocity. At shorter distances, agreement between the measurements and the numerical results is reasonably good. In the more distant region, corresponding to times subsequent to breakdown of the numerical solution, the measured amplitudes are reduced.

The breakdown of the numerical solution might be the result of a physical instability 


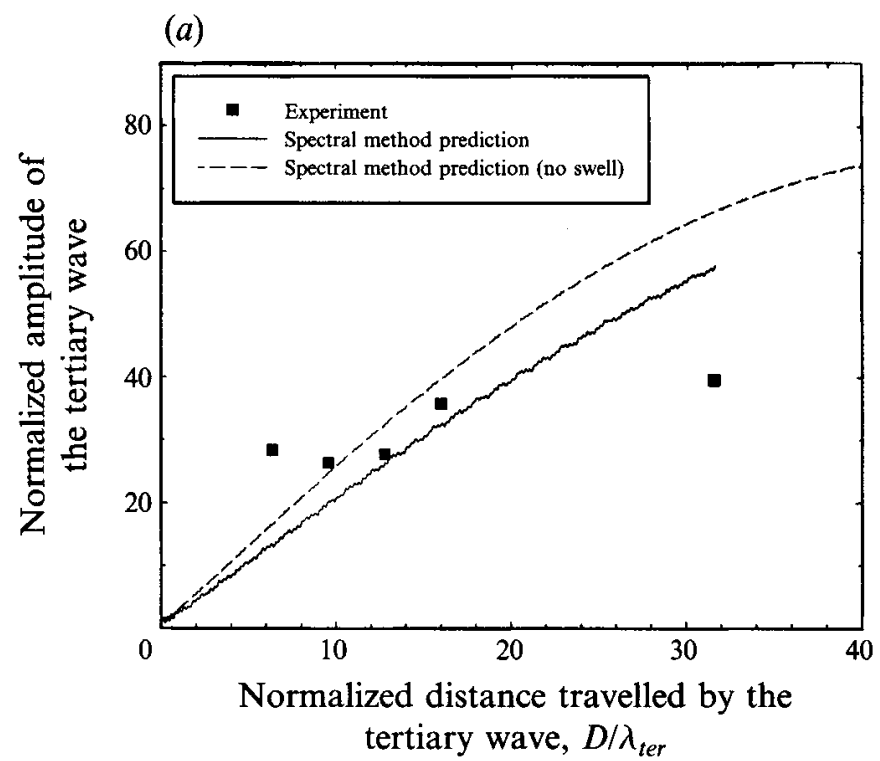

(b)

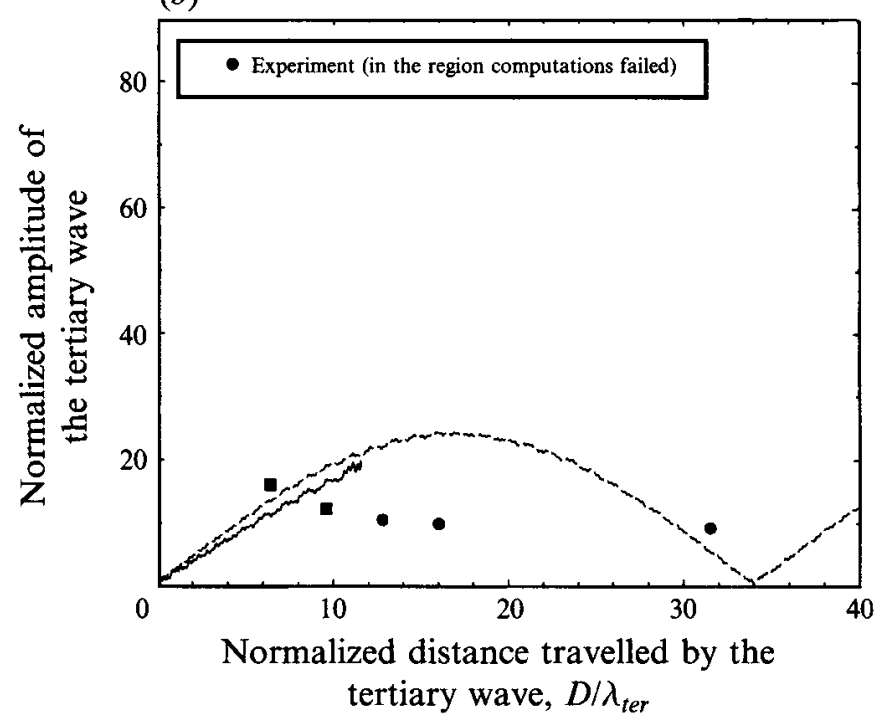

FIGURE 6. (a) A comparison of the spectral method with experiment for primary waves of steepness (a) 0.1 and (b) 0.174 and 0.18 , in the presence of an underlying long wave of steepness 0.05 .

associated with the combination of the swell and higher amplitudes of the primary waves. To test this possibility, we performed a two-dimensional BIEM calculation for the combination of the long primary wave and the underlying swell. We know (Ölmez 1991) that our BIEM calculations can be taken very nearly to the point of physical instability and by taking a two-dimensional case, our computational resources allowed a long enough run. The BIEM computations broke down when used with the same spatial resolution and filtering parameters as in the three-dimensional case.

The interaction of the swell with the short primary wave induces a second-order bound component at a frequency of $2 \omega_{1}-2 \omega_{3}$. As the wavelength of the swell was four times the wavelength of the long primary, the circular frequency of the swell turns out 


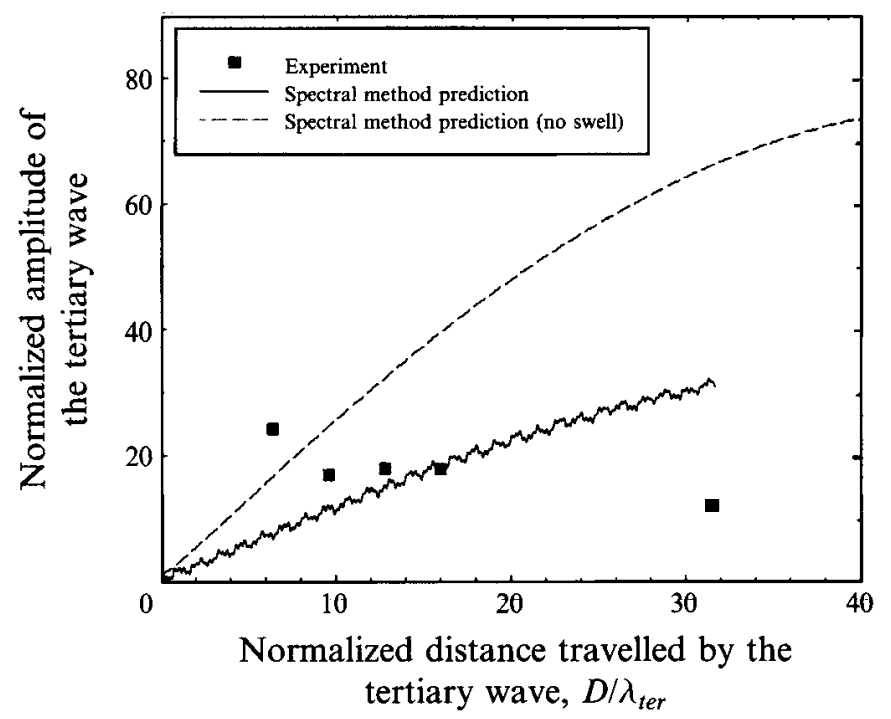

FIGURE 7. A comparison of the spectral method with experiment for primary waves of steepness 0.1 in the presence of an underlying long wave of steepness 0.075 .

to be twice the frequency of the long primary. This makes the frequency of the secondorder bound component the same as the frequency of the free-travelling tertiary wave. We calculated the magnitude of this bound component based on the third-order perturbation approximation. Although its contribution to the amplitude of the tertiary wave was found to be rather insignificant, we repeated the experiment with a different wavelength for swell that does not induce a bound component at the frequency of the tertiary. We selected a swell that has a wavelength of $5 \lambda_{2}$ with a higher steepness of 0.075 . This would have a more profound influence on the tertiary wave growth. We kept the primary wave steepnesses at 0.1 .

Figure 7 shows the amplitude of the tertiary wave as measured at the five stations and compares it with the results of the spectral method. Spectral method results for the absence of the long wave are also shown for comparison. As expected, the reduction in the tertiary wave growth is more pronounced owing to the presence of a steeper swell. Again, the spectral method is in good qualitative agreement with the observed amplitudes for the three middle points. The first and last points have amplitudes that are different from the computations by 1.7 and $1.9 \mathrm{~mm}$ respectively. The experimental results are consistent with our numerical findings that the presence of a long underlying wave strongly influences the growth of the tertiary wave. In the last two sections, we consider the mechanism that leads to a reduction in the tertiary wave growth and discuss its implications.

\section{Interactions leading to the reduction in tertiary wave growth in the presence of the long wave}

In Ölmez (1991), the perturbation expansion up to third order is given for the interacting triad in the presence of the long wave. Although the purpose of this expansion was to set initial conditions for the subsequent computations, we can use it here to qualitatively interpret why the presence of the long wave reduces the growth of the tertiary wave. A close examination of the third-order interaction terms reveals that some of them have the same length and frequency as the first-order primary wave 


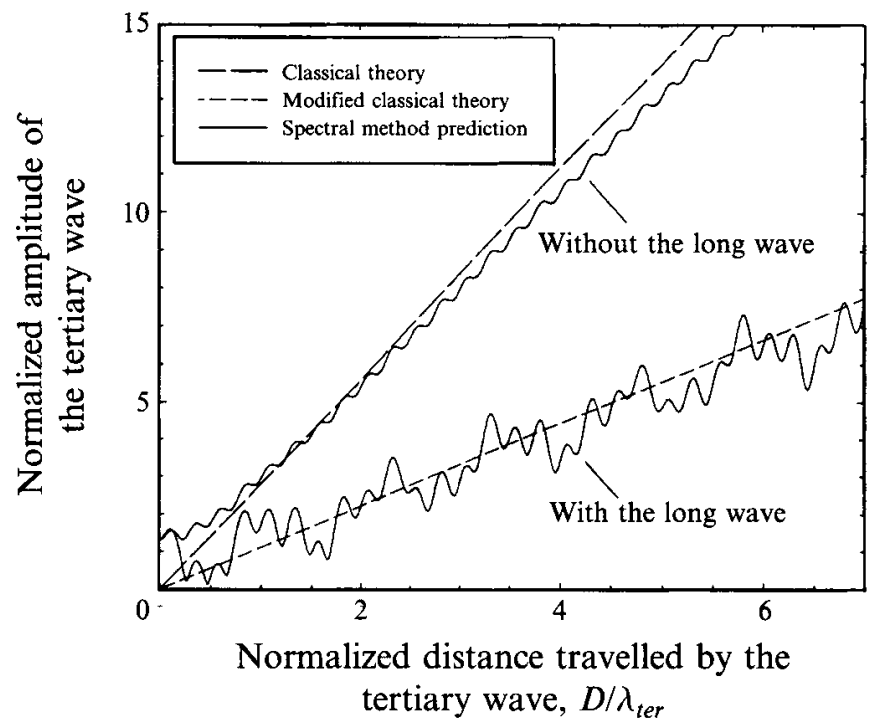

FIGURE 8. A comparison of the perturbation theory (based on altered amplitudes) with the spectral method in the absence and presence of the underlying long wave.

components. As a result, the sum of the elevations of the first- and third-order components of the surface elevation of the $n$th primary wave $(n=1,2)$ has the form

$$
\begin{array}{r}
\zeta_{n}^{(1)}+\zeta_{n}^{(3)}=\left[a_{n}+A_{n, 3}^{(3)}\left(a_{n}, a_{3}, k_{n}, k_{3}\right)+A_{n, m}^{(3)}\left(a_{n}, a_{m}, k_{n}, k_{m}\right)\right. \\
\left.+A_{n, n}^{(3)}\left(a_{n}, k_{n}\right)\right] \cos \left(k_{n} x-\omega_{n} t\right),
\end{array}
$$

where $a_{n}$ is the first-order amplitude and $m=1$ when $n=2$ and vice versa. The superscripts in parentheses denote the order. The $A_{n, 3}$ term is due to mutual interactions of the $n$th primary wave with the long wave, the $A_{n, m}$ term is due to mutual interactions of the $n$th primary wave with the $m$ th primary, and the $A_{n, n}$ term is due to interactions of the $n$th primary with itself (Stokes effect). The correction to the firstorder amplitude of a wave $a_{i}\left(k_{i}\right)$ due to its third-order interaction with another wave $a_{j}\left(k_{j}\right)$ can be expressed as

$$
A_{i j}^{(3)}=a_{i} a_{j}^{2} C\left(k_{i}, k_{j}, \theta\right)
$$

where $\theta$ is the angle between the wave components. When the wave directions are within $90^{\circ}$ of each other, the coupling coefficient, $C$, is always negative (Ölmez 1991) so it represents a reduction in wave amplitude.

The interaction is quite significant for the conditions of our experiments and numerical studies. For example, when all wave steepnesses are 0.1 , the amplitude reduction of the first primary wave is $37 \%$. This results in a reduction in the tertiary wave growth by about $60 \%$. This relatively large effect occurs because $a_{3} k_{1}$ is not small. When we examine the velocity potential of the first primary, we find a similar reduction in its component having the phase $\left(k_{n} x-\omega_{n} t\right)$. The combined first- and third-order wave amplitude and the velocity potential components with this phase satisfy the linear free-surface boundary conditions to within $4 \%$. The implication is that the combined first- and third-order primary wave 'looks like' a smaller linear wave due to third-order interactions with the long wave. When we evaluate the theoretical growth rate from the classical theory (Longuet-Higgins 1962) using the reduced amplitudes of the primary waves in lieu of the first-order components, the result of this 'prescription' is in good agreement with the spectral method prediction as shown in figure 8 . 

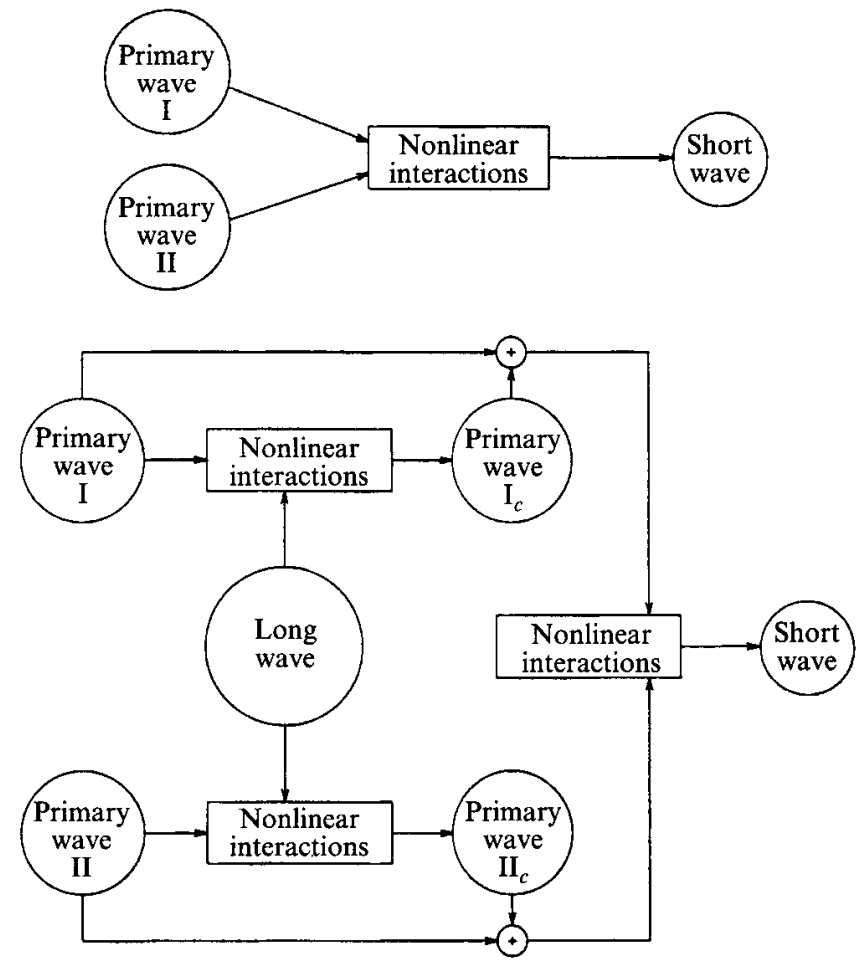

FIGURE 9. Block diagram of nonlinear interactions of the special triad with and without the presence of the long wave. Circles represent wave components. Boxes represent nonlinear interactions.

The important interactions, both with and without the presence of the long wave are indicated diagramatically in figure 9. The upper part of the figure represents the interaction for the special triad alone. The primary waves interact nonlinearly to transfer energy to the tertiary wave. The lower part of the figure, which includes the presence of the long wave, is more complicated. Nonlinear interactions between the long wave and each primary wave generate a third-order wave having the same lengths and frequencies as the primary waves, but of opposite phase. They are indicated by $\mathrm{I}_{c}$ and II $_{c}$ in figure 9 . The elevation and velocity potential of each of these third-order components very nearly satisfy the linear free-surface boundary condition. Thus when these third-order components are added to their respectively first-order primary components, the results are very nearly the same as linear components of reduced amplitudes. The reduction is a significant fraction of the linear wave amplitude because the long-wave amplitude is so much greater than the amplitudes of the primaries. The same physical nonlinear interaction that exists for the special triad alone exists for the waves whose amplitudes have been reduced by third-order interactions with the long wave and this results in less nonlinear energy transfer to the tertiary wave than occurs in the absence of the long wave.

An alternative, although entirely complementary, explanation has been suggested to us by Professor Owen Phillips. The long wave modulates the amplitudes, frequencies and wavenumbers of the primary waves. Because of the frequency and wavenumber modulations, parts of the primary waves go in and out of resonance with only the inresonance part contributing to the energy transfer. The explanations are related because the modulated primary waves are composed of components with reduced amplitudes at the initial frequencies and wavenumbers and sidebands at the sum and 
difference frequencies and wavenumbers. Only the components at the original frequencies and wavenumbers satisfy the resonance condition and contribute to the resonant interaction. This relationship is demonstrated in the growth rates shown in the examples. They contain a component with a period equivalent to 1.23 tertiary wavelengths which is the relative period between the long wave and the first primary. The growth rates go up and down as the system goes into and out of resonance at this period, with the mean rate of growth corresponding to the third-order-modified resonating components.

\section{Implications for calculation of energy transfer to the high-frequency tail of a wave spectrum}

The accepted method for calculating nonlinear energy transfer between frequency (or wavenumber) components of a gravity wave spectrum is based on the quartet interactions, but considered in a stochastic way. This is the method pioneered by Hasselmann (1962). The 'input' wave information is based on the linear components of the wave amplitudes. From the findings here, we would expect the calculated energy transfer to the high-frequency tail, based on resonant quartet interactions among linear wave components, to be an overestimate due to the presence of large long waves that are not part of the quartet. However, if the 'input components' are taken as the linear components plus the third-order amplitude modifications due to each component interacting with all the others, the estimate should be better. This issue has not been raised for most studies of nonlinear energy transfer because they deal mainly with the energy-containing portion of the spectrum, not the high-frequency tail. The latter is the part that exists 'on top of' much larger and longer waves.

When one measures ocean waves and separates the measurement into frequency or wavenumber components, each one corresponds to the complete component, not just the linear one. To calculate an estimate of the energy transfer distribution accurately in the high-frequency tail, it is desirable to know the linear and third-order components. This has led us to look into the relative sizes of the linear part of the wave spectrum, $\Phi_{11}$, which is $O\left(A^{2}\right)$ and the quadratic part of the spectrum, $\Phi_{22}+\Phi_{13}$ which is $O\left(A^{4}\right) . A$ is the linear wave amplitude. $\Phi_{22}$ results from products of second-order wave amplitudes and $\Phi_{13}$ results from products of first- and third-order wave amplitudes. Our development is described in the Appendix.

Using the results derived in the Appendix, here we evaluate the quadratic corrections to a unidirectional sea spectrum whose linear components are taken from the spectrum model of Bjerkaas \& Riedel (1979). This is not done for quantitative energy transfer calculations, which require a multi-directional wave field, but rather to demonstrate the importance of the higher-order contributions. One can argue, convincingly, that the high-frequency tail of the model spectrum contains all the higher-order corrections. However, using the model results as the linear spectrum suffices to show the importance of including the third-order amplitude effects when doing resonant energy transfer calculations, and of 'weeding out' the second-order influence which exists in measured spectra, when determining the first- and third-order amplitudes to be included. The important third-order component propagates at the same celerity as the linear component, but the second-order component at the same frequency has only half the celerity so it does not participate in the same resonant energy transfer interactions.

We carried out the quadratic spectrum calculations for two wind conditions: (i) a $19.5 \mathrm{~m}$ wind speed of $5 \mathrm{~m} \mathrm{~s}^{-1}$ with a friction velocity of $u_{*}=0.163 \mathrm{~m} \mathrm{~s}^{-1}$, and (ii) a $19.5 \mathrm{~m}$ wind speed of $10 \mathrm{~m} \mathrm{~s}^{-1}$ with a friction velocity of $0.362 \mathrm{~m} \mathrm{~s}^{-1}$. The friction 


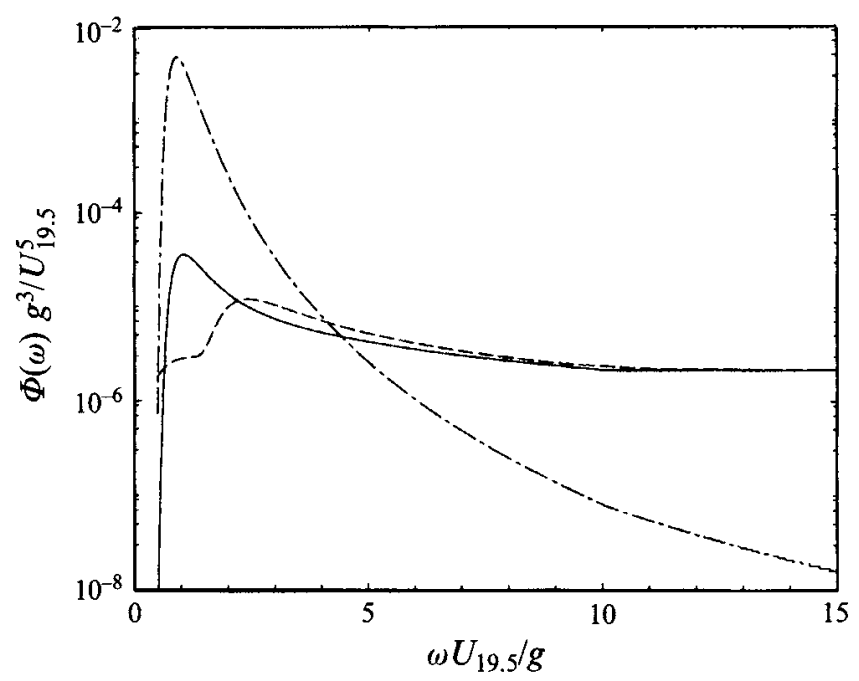

FIGURE 10. One-sided non-dimensionalized Bjerkaas-Riedel spectra: - - , linear spectrum $\Phi_{11}$; - , quadratic spectrum $\left|\Phi_{13}\right|$ (values are negative); ----, quadratic spectrum $\Phi_{22}$.

velocities were chosen by the methods given in Bjerkaas \& Riedel (1979). Since $u_{*} / U_{19.5}$ differs for the two calculations, the resulting spectra need not obey a similarity law. Nevertheless, when they are plotted in the non-dimensional form of figure 10 they are indistinguishable from each other. $\Phi_{13}$ dominates $\Phi_{22}$ in the frequency range of 0.5 to 2.0 times the spectral peak frequency. It is frequencies above this range that are of interest for our purposes here. There, the two quadratic spectral corrections are of similar magnitude, with $\left|\Phi_{22}\right|$ slightly greater than $\left|\Phi_{13}\right|$, but of opposite sign. For frequencies above about 4.5 times that of the spectral peak, the quadratic corrections to the spectrum exceed the ordinary linear spectrum so there the effects of still higherorder corrections might be important.

For resonant energy transfer interactions in which the energy-providing waves have frequencies less than 4.5 times that of the spectral peak, it is expected that the Hasselmann theory will be most accurate if wave amplitudes corresponding to $\Phi_{11}$ and $\Phi_{13}$ are used. The $\Phi_{13}$ correction becomes important for frequencies higher than 2.5 times that of the spectral peak. When one measures the spectrum there appear to be two methods for separating out $\Phi_{22}$, which needs to be done to obtain the sum of $\Phi_{11}$ and $\Phi_{13}$. One is to calculate all the components by methods similar to those given in the Appendix. This requires first extending the theory to the directional spectrum case. Alternatively, if the frequency-wavenumber spectrum is measured, $\Phi_{22}$ can be determined through data analysis. The reason is that both $\Phi_{11}$ and $\Phi_{13}$ will fall on the first-order dispersion line, $\omega^{2}=g|k|$, whereas $\Phi_{22}$ will not.

\section{Discussion and conclusions}

The principal finding of this paper is that when a resonantly interacting quartet, or in particular the special triad studied here, exists on top of a large long underlying wave, the energy transfer in the resonantly interacting group is diminished from its value in the absence of the long wave. This was demonstrated, both numerically and experimentally, when the long wave propagated in the same direction as one of the 'energy providing' interacting waves called 'primaries'. For the interacting special 
triad, when a long wave of amplitude $a_{3}$ and length about 25 times that of the growing tertiary wave, with $a_{3} k_{t e r} \approx 2.5$, was present the tertiary wave growth rate was reduced theoretically to about $60 \%$ of its value in the absence of the long wave. The physical reason for this is that the nonlinear interaction between the long wave and the primary reduces the primary wave amplitude. Mathematically, this is a third-order interaction depending linearly on the primary wave amplitude and quadratically on the long-wave amplitude. Although our studies here were limited to a primary and a long wave propagating in the same direction, use of the interaction coefficients given in Ölmez (1991) shows that when the two propagation directions are within $90^{\circ}$ of each other, the primary wave amplitude is reduced.

The findings are of importance for calculating energy transfer to the high-frequency tail, and for that matter to the higher frequency portion of the 'equilibrium range' of measured or modelled wave spectra. The reason for this is that the measured spectra are influenced not only by the first-order wave amplitudes, but also by higher-order components, particularly for the high frequencies and wavenumbers. Our results suggest that the classical resonant energy transfer formulae should use the combined first- and third-order wave components as inputs to maximize high-frequency accuracy. Our study of the quadratic correction to the wave spectrum shows effects with similar orders of magnitude from first-third-order contributions and second-second-order contributions. These can be separated on the basis of theory or by data analysis if the frequency-wavenumber spectrum is known.

At wave frequencies higher than about 4.5 times that of the spectral peak, the magnitudes of the quadratic spectrum corrections exceed the magnitude of the spectrum resulting from linear wave components only. At these high frequencies, even higher-order corrections could be dominant.

A second finding is that numerical spectral methods for computing interactions between waves of different amplitude and wavenumber scales $\left(a_{1}, k_{1}\right)$ and $\left(a_{2}, k_{2}\right)$, can be accurate even when $a_{2} k_{1}$ is not small in comparison to 1 . Results of the numerical spectral method are virtually identical to those of our fully nonlinear direct calculation in which the Green function integral equation is solved at every time step. The same reduction in tertiary-wave growth rate due to the long wave was found with our direct fully nonlinear method and by a spectral method which is based on perturbation theory, but which includes many interacting bound and free wave modes and which can accommodate an arbitrary order in the expansion (we used all orders up to and including the fifth). Evidently, the spectral method can be accurate for rather large values of $a_{2} k_{1}$ if a sufficiently large number of modes and orders are retained.

Our experimental measurements are in reasonably good agreement with the numerical predictions. There is no systematic difference and the scatter in the experimental data suggests that differences between the numerical and experimental results are due mostly to experimental error.

This research was supported by The Office of Naval Research (Contract N00014-89J-1185) and The National Science Foundation (Grant OCE-9216788). Computations were made on a VAX 9000 at MIT; on a Cray-2 at the MIT Supercomputer Facility; and on a Cray Y-MP at the Pittsburgh Supercomputing Center which is supported by The National Science Foundation (Grant OCE-900004P). The cooperation and assistance of the staff of the Institute for Marine Dynamics of the National Research Council of Canada is gratefully acknowledged. 


\section{Appendix. Quadratic correction to the linear wave spectrum}

At frequencies several times as large as that of the spectral peak, higher-order components of wave elevation make contributions to the wave spectrum that are significant in comparison to contributions from the linear components. At the very least, one needs to know the linear contributions to the spectrum to calculate energy transfer, and it is quite likely that improved estimates can be achieved if the contributions from third-order amplitude corrections are included. However, the quadratic correction to the wave spectrum includes, as we shall see here, the effects of both second- and third-order contributions to the wave elevation which have the same orders of magnitude. Tick (1959) first derived formulae for the influence of the secondorder contributions. Here we look at the problem in a more systematic way which yields the entire quadratic correction to the elevation spectrum including the equally important effects of second- and third-order wave elevations. Our development is described rather briefly here since as it follows along the lines used by Sclavounos (1922) for determining the quadratic correction for the spectrum of wave pressure which can be referred to for more details.

As is usual, the velocity potential, $\phi$, and the surface elevation, $\zeta$, are expanded in orders of the wave amplitude, with subscripts referring to order:

$$
\phi=\phi_{1}+\phi_{2}+\phi_{3}+\ldots, \quad \zeta=\zeta_{1}+\zeta_{2}+\zeta_{3}+\ldots
$$

Each of the velocity potentials, $\phi_{i}$, satisfies the Laplace equation.

The free-surface boundary conditions expanded up to third-order (Longuet-Higgins 1962) are

and

$$
g \zeta=-\left[\frac{\partial \phi}{\partial t}+\zeta \frac{\partial^{2} \phi}{\partial z \partial t}+\frac{1}{2} \zeta^{2} \frac{\partial^{3} \phi}{\partial z^{2} \partial t}+\ldots\right]-\left[\frac{1}{2} \nabla \phi \cdot \nabla \phi+\zeta \frac{\partial}{\partial z}\left(\frac{1}{2} \nabla \phi \cdot \nabla \phi\right)+\ldots\right],
$$

$$
\begin{aligned}
-\left(\frac{\partial^{2} \phi}{\partial t^{2}}+g \frac{\partial \phi}{\partial z}\right)= & {\left[\zeta \frac{\partial}{\partial z}\left(\frac{\partial^{2} \phi}{\partial t^{2}}+g \frac{\partial \phi}{\partial z}\right)+\frac{1}{2} \zeta^{2} \frac{\partial^{2}}{\partial z^{2}}\left(\frac{\partial^{2} \phi}{\partial t^{2}}+g \frac{\partial \phi}{\partial z}\right)+\ldots\right] } \\
& +\left[\frac{\partial}{\partial t}(\boldsymbol{\nabla} \phi \cdot \nabla \phi)+\zeta \frac{\partial^{2}}{\partial z \partial t}(\boldsymbol{\nabla} \phi \cdot \nabla \phi)+\ldots\right]+\left[\frac{1}{2} \nabla \phi \cdot \nabla(\nabla \phi \cdot \nabla \phi)+\ldots\right]
\end{aligned}
$$

which are satisfied on the quiescent free surface $z=0$. In this appendix $\nabla=(\partial / \partial x$, $\partial / \partial y, \partial / \partial z)$.

The first-order velocity potential has the form

$$
\phi_{1}(x, z, t)=\int_{\sigma} B(\sigma) \mathrm{e}^{|k| z} \mathrm{e}^{\mathrm{i}(k x-\sigma t)} \mathrm{d} \sigma
$$

where $\sigma$ is the circular frequency. For a random sea, $B(\sigma)$ is a Gaussian-distributed random variable with zero mean at each value of $\sigma$ (Kinsman 1984, chapter 7.3; Sclavounos 1992). The first-order components of the boundary conditions in (A 2) and (A 3), and our presumption of positive- $x$ travelling waves require that

$$
k=\sigma|\sigma| / g .
$$

The first-order component of the boundary condition (A 2) gives the first-order surface elevation directly as

where $A(\sigma)=\mathrm{i} \sigma B(\sigma) / g$.

$$
\zeta_{1}(x, t)=\int_{\sigma} A(\sigma) \mathrm{e}^{\mathrm{i}(k x-\sigma t)} \mathrm{d} \sigma,
$$


The second-order component of (A 3) is

$$
-\left(\frac{\partial^{2} \phi_{2}}{\partial t^{2}}+g \frac{\partial \phi_{2}}{\partial z}\right)=\zeta_{1} \frac{\partial}{\partial z}\left(\frac{\partial^{2} \phi_{1}}{\partial t^{2}}+g \frac{\partial \phi_{1}}{\partial z}\right)+\frac{\partial}{\partial t}\left(\nabla \phi_{1} \cdot \nabla \phi_{1}\right)
$$

which is to be satisfied on $z=0$. The solution is

$$
\phi_{2}(x, z, t)=\iint B\left(\sigma_{1}\right) B\left(\sigma_{2}\right) F_{2}\left(\sigma_{1}, \sigma_{2}\right) \mathrm{e}^{\left|k_{1}+k_{2}\right| z+\mathrm{i}\left[\left(k_{1}+k_{2}\right) x-\left(\sigma_{1}+\sigma_{2}\right) t\right]} \mathrm{d} \sigma_{1} \mathrm{~d} \sigma_{2},
$$

where kernel $F_{2}\left(\sigma_{1}, \sigma_{2}\right)$ is

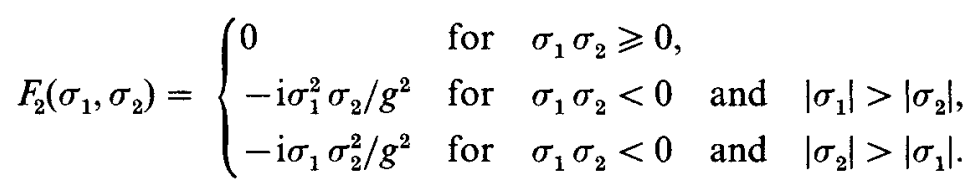

Understanding (A 9) requires remembering that each wave component at the physical frequency $\sigma$ contributes to the integrands at both $\sigma$ and $-\sigma$. The second-order component of (A 2) is

$$
g \zeta_{2}=-\frac{\partial \phi_{2}}{\partial t}-\zeta_{1} \frac{\partial^{2} \phi_{1}}{\partial z \partial t}-\frac{1}{2}\left(\nabla \phi_{1} \cdot \nabla \phi_{1}\right)
$$

which is to be satisfied on $z=0$. Substituting the first- and second-order potentials in (A 10) provides the expression for the second-order wave elevation:

$$
\zeta_{2}(x, t)=\iint A\left(\sigma_{1}\right) A\left(\sigma_{2}\right) Z_{2}\left(\sigma_{1}, \sigma_{2}\right) \mathrm{e}^{\mathrm{i}\left[\left(k_{1}+k_{2}\right) x-\left(\sigma_{1}+\sigma_{2}\right) t\right]} \mathrm{d} \sigma_{1} \mathrm{~d} \sigma_{2},
$$

where

$$
Z_{2}\left(\sigma_{1}, \sigma_{1}\right)=\frac{g}{\sigma_{1} \sigma_{2}}\left\{-\mathrm{i}\left(\sigma_{1}+\sigma_{2}\right) F_{2}\left(\sigma_{1}, \sigma_{2}\right)+\frac{\sigma_{1} \sigma_{2}}{g}\left|k_{2}\right|+\frac{1}{2}\left(\left|k_{1}\right|\left|k_{2}\right|-k_{1} k_{2}\right)\right\} .
$$

The third-order component of (A 3) is

$$
\begin{aligned}
-\left(\frac{\partial^{2} \phi_{3}}{\partial t^{2}}+g \frac{\partial \phi_{3}}{\partial z}\right)= & \zeta_{1} \frac{\partial}{\partial z}\left(\frac{\partial^{2} \phi_{2}}{\partial t^{2}}+g \frac{\partial \phi_{2}}{\partial z}\right)+\zeta_{2} \frac{\partial}{\partial z}\left(\frac{\partial^{2} \phi_{1}}{\partial t^{2}}+g \frac{\partial \phi_{1}}{\partial z}\right)+\frac{1}{2} \zeta_{1}^{2} \frac{\partial^{2}}{\partial z^{2}}\left(\frac{\partial^{2} \phi_{1}}{\partial t^{2}}+g \frac{\partial \phi_{1}}{\partial z}\right) \\
& +2 \frac{\partial}{\partial t}\left(\nabla \phi_{1} \cdot \nabla \phi_{2}\right)+\zeta_{1} \frac{\partial^{2}}{\partial z \partial t}\left(\nabla \phi_{1} \cdot \nabla \phi_{1}\right)+\frac{1}{2} \nabla \phi_{1} \cdot \nabla\left(\nabla \phi_{1} \cdot \nabla \phi_{1}\right), \quad(A
\end{aligned}
$$

which is to be satisfied on $z=0$. Using the linear and second-order solutions obtained earlier, this leads to the following form of the third-order potential:

$$
\phi_{3}(x, z, t)=\oint f f B\left(\sigma_{1}\right) B\left(\sigma_{2}\right) B\left(\sigma_{3}\right) F_{3}\left(\sigma_{1}, \sigma_{2}, \sigma_{3}\right)
$$

$$
\times \mathrm{e}^{\left|k_{1}+k_{2}+k_{3}\right| z+\mathrm{i}\left(\left[\left(k_{1}+k_{2}+k_{3}\right) x-\left(\sigma_{1}+\sigma_{2}+\sigma_{3}\right) t\right]\right.} \mathrm{d} \sigma_{1} \mathrm{~d} \sigma_{2} \mathrm{~d} \sigma_{3},
$$

where kernel $F_{3}\left(\sigma_{1}, \sigma_{2}, \sigma_{3}\right)$ is

$$
\begin{aligned}
F_{3}\left(\sigma_{1}, \sigma_{2}, \sigma_{3}\right)= & \frac{1}{\sigma^{2}-\sigma_{0}^{2}}\left\{\frac{\sigma_{1}}{g}\left(\sigma_{2}+\sigma_{3}\right)\left(\left|k_{2} k_{3}\right|-k_{2} k_{3}\right)\left(\left|k_{2}+k_{3}\right|-\left|k_{2}\right|-\left|k_{3}\right|\right)\right. \\
& +2 \mathrm{i}\left[\left|k_{1}\right|\left|k_{2}+k_{3}\right|-k_{1}\left(k_{2}+k_{3}\right)\right]\left(\sigma_{1}+\sigma_{2}+\sigma_{3}\right) F_{2}\left(\sigma_{2}, \sigma_{3}\right) \\
& \left.-\frac{1}{2}\left[\left|k_{1}\right|\left(\left|k_{2}\right|+\left|k_{3}\right|\right)-k_{1}\left(k_{2}+k_{3}\right)\right]\left(\left|k_{2} k_{3}\right|-k_{2} k_{3}\right)\right\},
\end{aligned}
$$


where $\sigma=\sigma_{1}+\sigma_{2}+\sigma_{3}$ and $\sigma_{0}^{2}=g\left|k_{1}+k_{2}+k_{3}\right|$. The Cauchy principal values appear in (A 14) because the apparent singularity at $\sigma^{2}=\sigma_{0}^{2}$ in (A 15) is to be replaced by a nonsingular secular form at this point. For the unidirectional travelling waves considered here, the secular term is out of phase with the linear wave component and does not contribute to the third-order potential (Sclavounos 1992) and is therefore properly neglected by the Cauchy principal value. The corresponding wave elevation can be found from (A 2):

$$
g \zeta_{3}=-\frac{\partial \phi_{3}}{\partial t}-\zeta_{1} \frac{\partial^{2} \phi_{2}}{\partial z \partial t}-\zeta_{2} \frac{\partial^{2} \phi_{1}}{\partial z \partial t}-\frac{1}{2} \zeta_{1}^{2} \frac{\partial^{3} \phi_{1}}{\partial z^{2} \partial t}-\left(\nabla \phi_{1} \cdot \nabla \phi_{2}\right)-\frac{1}{2} \zeta_{1} \frac{\partial}{\partial z}\left(\nabla \phi_{1} \cdot \nabla \phi_{1}\right)
$$

which leads to

$$
\zeta_{3}(x, t)=\int f f A\left(\sigma_{1}\right) A\left(\sigma_{2}\right) A\left(\sigma_{3}\right) Z_{3}\left(\sigma_{1}, \sigma_{2}, \sigma_{3}\right) \mathrm{e}^{\mathrm{i}\left[\left(k_{1}+k_{2}+k_{3}\right) x-\left(\sigma_{1}+\sigma_{2}+\sigma_{3}\right) t\right]} \mathrm{d} \sigma_{1} \mathrm{~d} \sigma_{2} \mathrm{~d} \sigma_{3},
$$

where

$$
\begin{aligned}
Z_{3}\left(\sigma_{1}, \sigma_{2}, \sigma_{3}\right)= & \frac{g^{2}}{\mathrm{i} \sigma_{1} \sigma_{2} \sigma_{3}}\left\{-\mathrm{i} \sigma F_{3}\left(\sigma_{1}, \sigma_{2}, \sigma_{3}\right)+\frac{\sigma_{1}\left(\sigma_{2}+\sigma_{3}\right)}{g} F_{2}\left(\sigma_{2}, \sigma_{3}\right)\left|k_{2}+k_{3}\right|\right. \\
& +\mathrm{i} \frac{\sigma_{1} \sigma_{2} \sigma_{3}}{g^{2}}\left[Z_{2}\left(\sigma_{1}, \sigma_{2}\right)\left|k_{3}\right|+\frac{1}{2} k_{3}^{2}\right]-\mathrm{i} \frac{\sigma_{1}}{g} k_{3}\left(k_{2}\left|k_{3}\right|-\left|k_{2}\right| k_{3}\right) \\
& \left.+F_{2}\left(\sigma_{2}, \sigma_{3}\right)\left[k_{1}\left|k_{2}+k_{3}\right|-\left|k_{1}\right|\left(k_{2}+k_{3}\right)\right]\right\} .
\end{aligned}
$$

The objective now is to determine the complete form of the quadratic spectrum. The autocorrelation function of the wave elevation is the real part of the ensemble average:

$$
R(t, \tau)=\operatorname{Re} E\left[\zeta(t) \zeta^{*}(t+\tau)\right]
$$

where $^{*}$ denotes the complex conjugate. The double-sided spectrum is

$$
\Phi(\omega, t)=\frac{1}{2 \pi} \int_{-\infty}^{+\infty} R(t, \tau) \mathrm{e}^{-\mathrm{i} \omega \tau} \mathrm{d} \tau .
$$

In terms of the elevation components up to third order:

$$
\begin{aligned}
R(t, \tau) & =\operatorname{Re}\left[E\left[\zeta_{1}(t) \zeta_{1}^{*}(t+\tau)\right]+E\left[\zeta_{2}(t) \zeta_{2}^{*}(t+\tau)\right]+E\left[\zeta_{1}(t) \zeta_{3}^{*}(t+\tau)+\zeta_{3}(t) \zeta_{1}^{*}(t+\tau)\right]+\ldots\right] \\
& =R_{11}(t, \tau)+R_{22}(t, \tau)+R_{13}(t, \tau)+\ldots,
\end{aligned}
$$

where the last two terms form the quadratic part of the autocorrelation function. Note that $E\left[\zeta_{1}(t) \zeta_{2}^{*}(t+\tau)\right]$ vanishes for a Gaussian process. The spectrum, including linear and quadratic components, will be obtained by taking the Fourier transform of (A 21), term by term, and is denoted as

$$
\Phi(\omega)=\Phi_{11}(\omega)+\Phi_{22}(\omega)+\Phi_{13}(\omega)+\ldots
$$

Using the expressions for the autocorrelation components (A 21) and for the secondorder surface elevation (A 11):

$$
\begin{array}{r}
R_{22}(t, \tau)=\operatorname{Re} \iiint \int E\left[A\left(\sigma_{1}\right) A\left(\sigma_{2}\right) A^{*}\left(\sigma_{3}\right) A^{*}\left(\sigma_{4}\right)\right] Z_{2}\left(\sigma_{1}, \sigma_{2}\right) Z_{2}^{*}\left(\sigma_{3}, \sigma_{4}\right) \\
\times \mathrm{e}^{-\mathrm{i}\left(\sigma_{1}+\sigma_{2}-\sigma_{3}-\sigma_{4}\right) t+\mathrm{i}\left(\sigma_{3}+\sigma_{4}\right) \tau} \mathrm{d} \sigma_{1} \mathrm{~d} \sigma_{2} \mathrm{~d} \sigma_{3} \mathrm{~d} \sigma_{4} .
\end{array}
$$

Since the $A$ are zero mean Gaussian variables and since (Kinsman 1984)

$$
E\left[A\left(\sigma_{1}\right) A^{*}\left(\sigma_{2}\right)\right]=S\left(\sigma_{1}\right) \delta\left(\sigma_{1}-\sigma_{2}\right)
$$


then

$$
\begin{aligned}
E\left[A\left(\sigma_{1}\right) A\left(\sigma_{2}\right) A^{*}\left(\sigma_{3}\right) A^{*}\left(\sigma_{4}\right)\right]= & E\left[A\left(\sigma_{1}\right) A\left(\sigma_{2}\right)\right] E\left[A^{*}\left(\sigma_{3}\right) A^{*}\left(\sigma_{4}\right)\right] \\
& +E\left[A\left(\sigma_{1}\right) A^{*}\left(\sigma_{3}\right)\right] E\left[A\left(\sigma_{2}\right) A^{*}\left(\sigma_{4}\right)\right] \\
& +E\left[A\left(\sigma_{1}\right) A^{*}\left(\sigma_{4}\right)\right] E\left[A\left(\sigma_{2}\right) A^{*}\left(\sigma_{3}\right)\right] \\
= & S\left(\sigma_{1}\right) S\left(\sigma_{3}\right) \delta\left(\sigma_{1}+\sigma_{2}\right) \delta\left(\sigma_{3}+\sigma_{4}\right) \\
& +S\left(\sigma_{1}\right) S\left(\sigma_{2}\right) \delta\left(\sigma_{1}-\sigma_{3}\right) \delta\left(\sigma_{2}-\sigma_{4}\right) \\
& +S\left(\sigma_{1}\right) S\left(\sigma_{2}\right) \delta\left(\sigma_{1}-\sigma_{4}\right) \delta\left(\sigma_{2}-\sigma_{3}\right) .
\end{aligned}
$$

This reduces (A 23) to

$$
\begin{aligned}
R_{22}(\tau)= & \iint S\left(\sigma_{1}\right) S\left(\sigma_{3}\right) Z_{2}\left(\sigma_{1},-\sigma_{1}\right) Z_{2}^{*}\left(\sigma_{3},-\sigma_{3}\right) \mathrm{d} \sigma_{1} \mathrm{~d} \sigma_{3} \\
& +\iint S\left(\sigma_{1}\right) S\left(\sigma_{2}\right) Z_{2}\left(\sigma_{1}, \sigma_{2}\right) Z_{2}^{*}\left(\sigma_{1}, \sigma_{2}\right) \mathrm{e}^{\mathrm{i}\left(\sigma_{1}+\sigma_{2}\right) \tau} \mathrm{d} \sigma_{1} \mathrm{~d} \sigma_{2} \\
& +\iint S\left(\sigma_{1}\right) S\left(\sigma_{2}\right) Z_{2}\left(\sigma_{1}, \sigma_{2}\right) Z_{2}^{*}\left(\sigma_{2}, \sigma_{1}\right) \mathrm{e}^{\mathrm{i}\left(\sigma_{1}+\sigma_{2}\right) \tau} \mathrm{d} \sigma_{1} \mathrm{~d} \sigma_{2}
\end{aligned}
$$

Carrying out the Fourier transform (A 20) the corresponding contribution to the quadratic spectrum is

$$
\begin{aligned}
\Phi_{22}(\omega)= & \delta(\omega) \iint S\left(\sigma_{1}\right) S\left(\sigma_{3}\right) Z_{2}\left(\sigma_{1},-\sigma_{1}\right) Z_{2}^{*}\left(\sigma_{3},-\sigma_{3}\right) \mathrm{d} \sigma_{1} \mathrm{~d} \sigma_{3} \\
& +2 \iint S\left(\sigma_{1}\right) S\left(\sigma_{2}\right) \delta\left(\sigma_{1}+\sigma_{2}-\omega\right) Z_{2}\left(\sigma_{1}, \sigma_{2}\right) Z_{2}^{*}\left(\sigma_{1}, \sigma_{2}\right) \mathrm{d} \sigma_{1} \mathrm{~d} \sigma_{2} .
\end{aligned}
$$

The first term on the right-hand side of (A 27) is due to non-zero mean value of the second-order wave elevation which is not of interest here and will be disregarded. The remaining term can be integrated once to give

$$
\Phi_{22}(\omega)=2 \int_{-\infty}^{+\infty} S(\sigma) S(\omega-\sigma) Z_{2}(\sigma, \omega-\sigma) Z_{2}^{*}(\sigma, \omega-\sigma) \mathrm{d} \sigma .
$$

It should be noted that contributions to $\Phi_{22}$ at frequency $\omega$ are due to products of wave components at different frequencies which sum to $\omega$.

Using (A 6), (A 17) and (A 21):

$$
\begin{aligned}
R_{13}(t, \tau)= & \operatorname{Re}\left[\iiint \int E\left[A\left(\sigma_{1}\right) A^{*}\left(\sigma_{2}\right) A^{*}\left(\sigma_{3}\right) A^{*}\left(\sigma_{4}\right)\right] Z_{3}^{*}\left(\sigma_{2}, \sigma_{3}, \sigma_{4}\right)\right. \\
& \times \mathrm{e}^{-\mathbf{i}\left(\sigma_{1}-\sigma_{2}-\sigma_{3}-\sigma_{4}\right) t+\mathrm{i}\left(\sigma_{2}+\sigma_{3}+\sigma_{4}\right) \tau} \mathrm{d} \sigma_{1} \mathrm{~d} \sigma_{2} \mathrm{~d} \sigma_{3} \mathrm{~d} \sigma_{4} \\
& +\iiint \int E\left[A^{*}\left(\sigma_{1}\right) A\left(\sigma_{2}\right) A\left(\sigma_{3}\right) A\left(\sigma_{4}\right)\right] Z_{3}\left(\sigma_{2}, \sigma_{3}, \sigma_{4}\right) \\
& \left.\times \mathrm{e}^{-\mathbf{i}\left(-\sigma_{1}+\sigma_{2}+\sigma_{3}+\sigma_{4}\right) t+\mathrm{i} \sigma_{1} \tau} \mathrm{d} \sigma_{1} \mathrm{~d} \sigma_{2} \mathrm{~d} \sigma_{3} \mathrm{~d} \sigma_{4}\right]
\end{aligned}
$$

which reduces to

$$
\begin{aligned}
R_{13}(\tau)= & \iint S\left(\sigma_{1}\right) S\left(\sigma_{3}\right) Z_{3}^{*}\left(\sigma_{1}, \sigma_{3},-\sigma_{3}\right) \mathrm{e}^{\mathrm{i} \sigma_{1} \tau} \mathrm{d} \sigma_{1} \mathrm{~d} \sigma_{3} \\
& +\iint S\left(\sigma_{1}\right) S\left(\sigma_{2}\right) Z_{3}^{*}\left(\sigma_{2}, \sigma_{1},-\sigma_{2}\right) \mathrm{e}^{\mathrm{i} \sigma_{1} \tau} \mathrm{d} \sigma_{1} \mathrm{~d} \sigma_{2} \\
& +\iint S\left(\sigma_{1}\right) S\left(\sigma_{2}\right) Z_{3}^{*}\left(\sigma_{2},-\sigma_{2}, \sigma_{1}\right) \mathrm{e}^{\mathrm{i} \sigma_{1} \tau} \mathrm{d} \sigma_{1} \mathrm{~d} \sigma_{2}
\end{aligned}
$$


This can be written as

$$
R_{13}(\tau)=\iint S\left(\sigma_{1}\right) S\left(\sigma_{2}\right)\left[G\left(\sigma_{1}, \sigma_{2},-\sigma_{2}\right)+G\left(\sigma_{2}, \sigma_{1},-\sigma_{2}\right)+G\left(\sigma_{2},-\sigma_{2}, \sigma_{1}\right)\right] \mathrm{e}^{\mathrm{i} \sigma_{1} \tau} \mathrm{d} \sigma_{1} \mathrm{~d} \sigma_{2}
$$

where

$$
G\left(\sigma_{1}, \sigma_{2}, \sigma_{3}\right)=Z_{3}\left(\sigma_{1}, \sigma_{2}, \sigma_{3}\right)+Z_{3}^{*}\left(\sigma_{1}, \sigma_{2}, \sigma_{3}\right) .
$$

By taking the Fourier transform of (A 31) and integrating over $\sigma_{1}$ :

$$
\Phi_{13}(\omega)=S(\omega) \int_{-\infty}^{+\infty} S(\sigma)[G(\omega, \sigma,-\sigma)+G(\sigma, \omega,-\sigma)+G(\sigma,-\sigma, \omega)] \mathrm{d} \sigma .
$$

The $\Phi_{13}$ spectrum at frequency $\omega$ is proportional to the linear spectrum, $\Phi_{11}$, at the same frequency $\omega$ and also to a weighted average of the entire $\Phi_{11}$ spectrum. For our unidirectional seas the weighting function is always negative.

Equations (A 28) and (A 33) together complete the quadratic part of the linear spectrum.

\section{REFERENCES}

Baker, G. R., Meiron, D. I. \& OrszaG, S. A. 1982 Generalized vortex methods for free-surface flow problems. J. Fluid Mech. 123, 477-501.

BENNEY, D. J. 1962 Non-linear gravity wave interactions. J. Fluid Mech. 12, 577-584.

BjerkaAs, A. W. \& Riedel, F. W. 1979 Proposed model for the elevation spectrum of a windroughened sea surface. Tech. Memo. The Johns Hopkins University, APL TG 1328, Laurel, Maryland 20810.

Crawford, D. R., Saffman, P. G. \& Yuen, H. C. 1980 Evolution of a random inhomogeneous field of nonlinear deep-water gravity waves. Wave Motion 2, 1-16.

Dommermuth, D. G. \& YUE, D. K. P. 1987 A high-order spectral method for the study of nonlinear gravity waves. J. Fluid Mech. 184, 267-288.

Dommermuth, D. G., Yue, D. K. P., Lin, W. M., Rapp, R. J., Chan, E. S. \& Melville, W. K. 1988 Deep-water plunging breakers: a comparison between potential theory and experiments. $J$. Fluid Mech. 189, 423-442.

HasselmanN, K. 1962 On the non-linear energy transfer in a gravity-wave spectrum. Part 1. General theory. J. Fluid Mech. 12, 481-500.

HasselmanN, S. \& HasselmanN, K. 1985 Computations and parameterization of the nonlinear energy transfer in a gravity-wave spectrum. Part I: A new method for efficient computations of the exact nonlinear transfer integral. J. Phys. Oceanogr. 15, 1369-1377.

JAMIESON, W. W. \& MANSARD, E. P. D. 1987 An efficient upright wave absorber. In ASCE Specialty Conference on Coastal Hydrodynamics, University of Delaware, Newark, Delaware, June 29-July 1, pp. 609-633.

Kinsman, B. 1984 Wind Waves, 2nd edn. Dover.

LONGUET-HigGins, M. S. 1962 Resonant interactions between two trains of gravity waves. J. Fluid Mech. 12, 321-332.

Longuet-Higgins, M.S. \& Cokelet, E. D. 1976. The deformation of steep surface waves on water, I. A numerical method of computation. Proc. R. Soc. Lond. A 350, 1-26.

Longuet-Higgins, M.S. \& SMith, N. D. 1966 An experiment on third-order resonant wave interactions. J. Fluid Mech. 25, 417-435.

McGoldrick, L. F., Phillips, O. M., Huang, N. E. \& Hodgson, T. H. 1966 Measurements of third-order resonant wave interactions. J. Fluid Mech. 25, 437-456.

Newman, J. N. 1986 Distribution of sources and normal dipoles over a quadrilateral panel. J. Engng Maths 20, 113-126.

ÖLMEZ, H. S. 1991 Numerical evaluation of nonlinear energy transfer to short gravity waves in the presence of long waves. ScD thesis, Massachusetts Institute of Technology. 
ÖlmEz, H.S. \& MilgRAM, J.H. 1992 An experimental study of attenuation of short waves by turbulence. J. Fluid Mech. 239, 133-156.

ÖLmEZ, H. S. \& MilgRAM, J. H. 1995 Numerical methods for nonlinear interactions between water waves. J. Comput. Phys. 18 (in press).

Phillips, O. M. 1960 On the dynamics of unsteady gravity waves of finite amplitude. Part 1. The elementary interactions. J. Fluid Mech. 9, 193-217.

SCLavounos, P. D. 1962 On the quadratic effect of random gravity waves on a vertical boundary. J. Fluid Mech. 242, 475-489.

STIASSNIE, M. \& SHEMER, L. 1984 On modifications of the Zakharov equation for surface gravity waves. J. Fluid Mech. 143, 47-67.

TICK, L. J. 1959 A non-linear random model of gravity waves. J. Maths Mech. 8, 643-652.

Tомта, H. 1989 Theoretical and experimental investigations of interaction among deep-water gravity waves. Ship Res. Inst. (Senpaku Gijutsu Kenkyujo Hokoko), 26, 1-100.

West, B. J., Brueckner, K. A., Janda, R. S., Milder, D. M. \& Milton, R. L. 1987 A new numerical method for surface hydrodynamics. J. Geophys. Res. 92, 11803-11824.

ZaKHAROv, V. E. 1968 Stability of periodic waves of finite amplitude on the surface of a deep fluid. J. Appl. Mech. Tech. Phys. (English Trans.), 9, 190-194. 